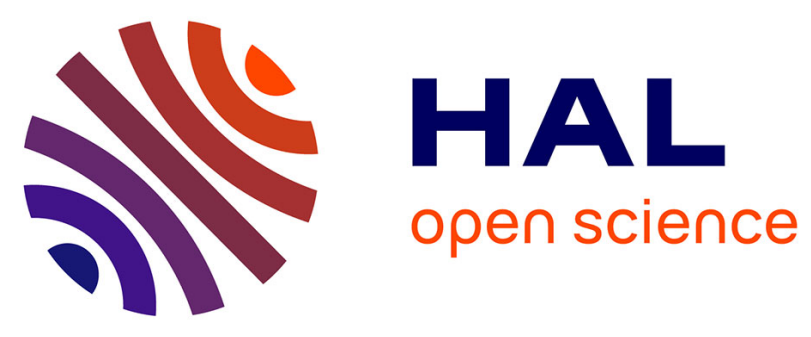

\title{
Estimating single-crystal elastic constants of polycrystalline $\beta$ metastable titanium alloy: A Bayesian inference analysis based on high energy X-ray diffraction and micromechanical modeling
}

Ravi Raj Purohit Purushottam Raj Purohit, Thiebaud Richeton, Stephane Berbenni, Lionel Germain, Nathalie Gey, Thomas Connolley, Olivier Castelnau

\section{- To cite this version:}

Ravi Raj Purohit Purushottam Raj Purohit, Thiebaud Richeton, Stephane Berbenni, Lionel Germain, Nathalie Gey, et al.. Estimating single-crystal elastic constants of polycrystalline $\beta$ metastable titanium alloy: A Bayesian inference analysis based on high energy X-ray diffraction and micromechanical modeling. Acta Materialia, 2021, 208, pp.116762. 10.1016/j.actamat.2021.116762 . hal-03156360

\section{HAL Id: hal-03156360 \\ https://hal.univ-lorraine.fr/hal-03156360}

Submitted on 8 Mar 2021

HAL is a multi-disciplinary open access archive for the deposit and dissemination of scientific research documents, whether they are published or not. The documents may come from teaching and research institutions in France or abroad, or from public or private research centers.
L'archive ouverte pluridisciplinaire HAL, est destinée au dépôt et à la diffusion de documents scientifiques de niveau recherche, publiés ou non, émanant des établissements d'enseignement et de recherche français ou étrangers, des laboratoires publics ou privés.

\section{(ㅇ)(1) $\$$}

Distributed under a Creative Commons Attribution - NonCommercial - NoDerivatives 44.0 


\title{
Estimating single-crystal elastic constants of polycrystalline $\beta$ metastable titanium alloy: A Bayesian inference analysis based on high energy X-ray diffraction and micromechanical modeling
}

\author{
Ravi Raj Purohit Purushottam Raj Purohita,b,c,*, Thiebaud Richeton ${ }^{\mathrm{a}, \mathrm{c}}$, Stephane Berbenni ${ }^{\mathrm{a}, \mathrm{c}}$, \\ Lionel Germain ${ }^{\mathrm{a}, \mathrm{c}}$, Nathalie Gey ${ }^{\mathrm{a}, \mathrm{c}}$, Thomas Connolley ${ }^{\mathrm{d}}$, Olivier Castelnau ${ }^{\mathrm{e}}$ \\ a Université de Lorraine, CNRS, Arts et Métiers Paris Tech, LEM3, F-57000 Metz, France \\ ${ }^{\mathrm{b}}$ Institut de Recherche Technologique Materiaux, Metallurgie, Procedes (IRT M2P), 4 Rue Augustin Fresnel, 57070 Metz, France \\ ${ }^{\mathrm{c}}$ Laboratory of Excellence on Design of Alloy Metals for Low-mAss Structures (DAMAS), Université de Lorraine, France \\ d Diamond Light Source, Oxfordshire, UK \\ e PIMM, Arts et Metiers Institute of Technology, CNRS, Cnam, HESAM University, 151 boulevard de l'Hopital, 75013 Paris France
}

Keywords:

Single-crystal elastic constants

Bayesian inference

Synchrotron XRD

Near $\beta$ titanium alloy

Elastic anisotropy

Elastic self-consistent modeling

\begin{abstract}
A B S T R A C T
A two-phase near- $\beta$ titanium alloy (Ti-10V-2Fe-3Al, or Ti-1023) in its as-forged state is employed to illustrate the feasibility of a Bayesian framework to identify single-crystal elastic constants (SEC). High Energy X-ray diffraction (HE-XRD) obtained at the Diamond synchrotron source are used to characterize the evolution of lattice strains for various grain orientations during in situ specimen loading in the elastic regime. On the other hand, specimen behavior and grain deformation are estimated using the elastic self-consistent (ELSC) homogenization scheme. The XRD data and micromechanical modelling are revisited with a Bayesian framework. The effect of different material parameters (crystallographic and morphological textures, phase volume fraction) of the micromechanical model and the biases introduced by the XRD data on the identification of the SEC of the $\beta$ phase are systematically investigated. In this respect, all the three cubic elastic constants of the $\beta$ phase $\left(C_{11}^{\beta}, C_{12}^{\beta}, C_{44}^{\beta}\right)$ in the Ti-1023 alloy have been derived with their uncertainties. The grain aspect ratio in the ELSC model, which is often not considered in the literature, is found to be an important parameter in affecting the identified SEC. The Bayesian inference suggests a high probability for non-spherical grains (aspect ratio of $\sim 3.8 \pm 0.8$ ) $: C_{11}^{\beta}=92.6 \pm 19.1 \mathrm{GPa}, C_{12}^{\beta}=82.5 \pm 16.3 \mathrm{GPa}, C_{44}^{\beta}=43.5 \pm 7.1 \mathrm{GPa}$. The uncertainty obtained by Bayesian approach lies in the range of $\sim 1-3 \mathrm{GPa}$ for the shear modulus $\mu^{\prime}=\frac{C_{11}^{\beta}-C_{12}^{\beta}}{2}$, and $\sim 7 \mathrm{GPa}$ for the shear modulus $\mu^{\prime \prime}=C_{44}^{\beta}$, while it is significantly larger in the case of the bulk modulus $\frac{C_{11}^{\beta}+2 C_{12}^{\beta}}{3}(\sim 17-24 \mathrm{GPa})$.
\end{abstract}

\section{Introduction}

Titanium and its alloys are attractive in the aeronautic industry due to their high strength to density ratios. The near- $\beta$ titanium alloy $\mathrm{Ti}-10 \mathrm{~V}-2 \mathrm{Fe}-3 \mathrm{Al}$ (Ti-1023) developed in the 1970s is often used in the forged pieces of aircraft for landing gears and rotor systems [1]. Its in-service microstructure is multiscale and complex. After forging, it is composed of hexagonal primary $\alpha_{\mathrm{p}}$ nodules (micrometric size) embedded in a cubic $\beta$ matrix with millimetric grains ( $\beta / \alpha$-forged state). Further aging partially transforms the $\beta$ matrix

\footnotetext{
* Corresponding author.

E-mail address: ravi-raj-purohit.purushottam-raj-purohit@univ-lorraine.fr (R.R.P. Purushottam Raj Purohit).
}

to secondary $\alpha_{\text {s }}$ platelets of nanometric size [2]. The alloy potentially exhibits remarkable mechanical behavior: yield stress up to $1200 \mathrm{MPa}$ with a ductility of $7 \%$. However, these properties are highly sensitive to local microstructure variations, which are not fully controlled in large forged pieces. In fact, the elastic properties of this alloy are far from being fully understood yet. At least, there is a reasonable agreement in the literature when it comes to the single-crystal elastic constants (SEC) of the $\alpha$ phase. However, for the $\beta$ (BCC) phase, the published SEC $C_{11}^{\beta}, C_{12}^{\beta}, C_{44}^{\beta}$ span very different values, refer to Table 1 . These SEC define three independent elastic moduli, the shear moduli, defined as $\mu^{\prime \prime}=\mathrm{C}_{44}^{\beta}$ and $\mu^{\prime}=\frac{c_{11}^{\beta}-c_{12}^{\beta}}{2}$ and the bulk modulus, $K=\frac{c_{11}^{\beta}+2 c_{12}^{\beta}}{3}$. The anisotropy ratio, $A=\frac{2 C_{44}^{\beta}}{C_{11}^{\beta}-C_{12}^{\beta}}=\frac{\mu^{\prime \prime}}{\mu^{\prime}}$ [3], is found to range over very large val- 
Table 1

Literature range of the three SEC of the $\beta$ phase.

\begin{tabular}{|c|c|c|c|c|c|c|c|}
\hline References & Material & $\mathrm{C}_{11}^{\beta}(\mathrm{GPa})$ & $\mathrm{C}_{12}^{\beta}(\mathrm{GPa})$ & $\mathrm{C}_{44}^{\beta}\left(\mu^{\prime \prime}\right)(\mathrm{GPa})$ & A & $\mu^{\prime}(\mathrm{GPa})$ & $\mathrm{K}(\mathrm{GPa})$ \\
\hline Fréour et al. [5] & Ti-17 & 174 & 116 & 41 & 1.4 & 29 & 135.3 \\
\hline Fréour et al. [6] & Ti-17 & 167 & 115 & 44 & 1.7 & 26 & 132.3 \\
\hline Nejezchlebová et al. [7] & LCB & 138 & 102.2 & 42.5 & 2.4 & 17.9 & 114.1 \\
\hline Martin [8] & Ti-5553 & 100 & 70 & 36 & 2.4 & 15 & 80.0 \\
\hline Petry et al. [9] & Pure & 134 & 110 & 36 & 3 & 12 & 118.0 \\
\hline Brandes and Brook [10] & Pure & 134 & 110 & 55 & 4.6 & 12 & 118.0 \\
\hline Fisher and Dever [11] & Pure & 99 & 85 & 33.6 & 4.8 & 7 & 89.7 \\
\hline Kim and Rokhlin [12] & Ti-6242 & 135 & 113 & 54.9 & 5 & 11 & 120.3 \\
\hline Ledbetter et al. [13] & Pure & 97.7 & 82.7 & 37.5 & 5 & 7.5 & 87.7 \\
\hline Raghunathan et al. [14] & Ti-1023 Forged & 140 & 128 & 50 & 8.3 & 6 & 132.0 \\
\hline Raghunathan et al. [14] & Ti-1023 Aged & 165.0 & 118.0 & 45.0 & 1.9 & 23.5 & 133.7 \\
\hline Meng et al. [15] & $\mathrm{Ti}-39 \mathrm{Nb}$ & 137.7 & 107.7 & 21.4 & 1.4 & 15 & 117.7 \\
\hline Talling et al. [16] & Ti-36Nb-2Ta-3Zr-0.30 & 125 & 93 & 28 & 1.8 & 16 & 103.7 \\
\hline Meng et al. [17] & $\mathrm{Ti}-36 \mathrm{Nb}-5 \mathrm{Zr}$ & 142.8 & 108.6 & 25.4 & 1.5 & 17.1 & 120.0 \\
\hline Obbard et al. [18] & Ti-24Nb-4Zr-8Sn & 54 & 33 & 32.5 & 3.1 & 10.5 & 40.0 \\
\hline Bhattacharyya et al. [19] & $\beta$-Timetal-18 & 118 & 79 & 39 & 2 & 19.5 & 92.0 \\
\hline Obbard et al. [20] & Ti-30Nb-10Ta-5Zr & 67.1 & 39.9 & 29.8 & 2.19 & 13.6 & 49 \\
\hline Tane et al. [21] & $\mathrm{Ti}-35.7 \mathrm{Nb}-2.1 \mathrm{Ta}-3.2 \mathrm{Zr}-0.420$ & 125 & 90 & 31 & 1.8 & 17.5 & 102 \\
\hline Jeong et al. [22] & $\mathrm{Ti}-40 \mathrm{Nb}$ & 141.3 & 117.0 & 32.1 & 2.7 & 12.1 & 125.1 \\
\hline
\end{tabular}

ues from 1.4 to 8 , see Table 1 . The differences are possibly due to chemical compositions of the $\beta$ phase, although one cannot sweep out the difficulties to measure local elastic constants in a multiphase polycrystalline specimen. Such lack of accuracy impedes a predictive understanding of the material's behavior. For example, in the study by Raghunathan et al [14], where the forged and aged state have been studied, the strong difference in the identified SEC for the $\beta$ phase is a possible result of microstructure as well as the chemical composition of the $\beta$ phase. A recent study [4] on polycrystalline Ti-6Al-2Sn-4Zr-6Mo alloy with X-ray diffraction reports a decrease in SEC of the $\beta$ phase by $10 \%$ (towards the single-phase alloy) when accounting for the load partitioning from the $\beta$ phase to the $\alpha$ phase, a feature that will be naturally taken into account with the Self-Consistent model used here.

Prior research on this alloy has been focused on ex-situ mechanical tests and micromechanical modeling to explain the elastic and viscoplastic properties $[23,24]$. Some studies have been carried out to quantify the elastic constants in the $\beta$-titanium alloys due to the strong relationship between the shear modulus $\left(\mu^{\prime}\right)$ and the stability of the phase [25]. Other studies have also focused on the correlation of shear modulus $\left(\mu^{\prime}\right)$ to the Young's modulus $[26,27]$. Recently, Lhadi et al [28] have shown that SEC corresponding to different anisotropy ratios (of 2.4,3, and 8.3) can all lead to the same effective Young moduli and similar macroscopic behavior in the elastic regime, but very different inter-granular stresses in the course of elasto-plastic deformation.

The standard SEC characterization involves experiments on large single crystals using classical methods such as ultrasonic wave velocities [29,30], rectangular parallelepiped resonance [31]. However, it is rather difficult to produce single crystals with exact composition to that of a phase within a multi-phase alloy. In the literature, several studies on single-phase microstructures have adopted an inverse approach using the elastic self-consistent model (ELSC) to identify the diffraction elastic moduli (DEM) from the X-ray diffraction (XRD) analysis and the macroscopic stiffness $[17,18,32,33]$. This can be challenging in the case of a multiphase alloy where the SEC of each phase is inherently different due to crystal symmetry. A variety of micromechanical models exist that take into account the crystallographic texture and grain morphology to establish the elastic properties of a polycrystal [34-38]. Given the computational simplicity, the two-phase interactions in the model are often approximated through an average procedure using an elastic homogeneous equivalent medium (HEM). The self- consistent scheme $[37,39,40]$ has also been successfully applied to study the load-partitioning in titanium alloys [14,18,33,41-44].

However, studies $[15,17,18]$ with inverse identification by the least-squares method have reported that the DEM for Ti-alloys can be insensitive to the bulk modulus, thus resulting in several possible SEC that satisfies the lattice strain evolution i.e. the associated uncertainties are rather large. In the literature $[17,18]$, this is addressed by evaluating the SEC for a fixed bulk modulus, which is a theoretical approximation. Recently, a study on $\beta$ Timetal-18 [19] has employed high energy X-ray diffraction and a micromechanical analysis of stress-strain inhomogeneities with a fast Fourier transform (FFT)-based formulation (MASSIF) to identify the SEC of the $\beta$ phase. A grain-to-grain analysis in the MASSIF framework suggested a strong effect on the elastic anisotropy, i.e., higher anisotropy ratio $(A=3.5)$ provided better correlation of all the strains components.

Part of the variability reported in Table 1 may come from chemical composition variations, and it is not the scope of the present paper to discuss this aspect. We want to stress that multiple SEC have been identified for the $\beta$ phase and most of the studies have used inverse identification and have only provided one unique value for the SEC of the $\beta$ phase without estimating the role of different microstructural parameters and none of them provide an uncertainty estimate on the identified value. In the current study, to quantify the uncertainties and also to study the effect of different material parameters on the SEC identification, a Bayesian framework combined with an inverse identification approach to probe a large $C_{\mathrm{ij}}$ space is employed. The Bayesian framework provides the advantage of exploring the large $\mathrm{Cij}$ space while generating a distribution of acceptable values. For example, such a framework has already been used in the context of spherical indentation measurements to identify the SEC for single-phase materials [4548].

The objective of this paper is to present the Bayesian framework in synergy with high-quality XRD data acquired during tensile loading at the DIAMOND synchrotron source and the ELSC model. This is applied to a near $\beta$ Ti-1023 alloy to estimate the uncertainties on the SEC of the cubic $(\beta)$ phase from XRD data. The combination of XRD and ELSC modeling is often employed in the literature to evaluate the SEC. However, to the best of authors' knowledge, this is the first time that these methods are employed in a Bayesian framework to quantify the uncertainties of SEC for a polycrystalline multi-phase alloy. Here, a Bayesian 
framework is elaborated together with the Metropolis-Hasting algorithm [49] and the ELSC model [37,38] to evaluate the experimental data, with a particular focus on XRD data evaluation. This framework is then applied to the experimental data (macroscopic stiffness and XRD lattice strains), and the results are discussed along with the literature. Different parameters in the ELSC micromechanical model are explored and the experimental errors influencing the quantification of uncertainties of the SEC are discussed.

\section{Methods}

\subsection{Material}

For our study, an $(\alpha+\beta)$ forged billet of $\beta$ metastable titanium alloy Ti-10V-2Fe-3Al (by wt. \%) was provided by Timet, France. The $\beta$ transus of this alloy is $795^{\circ} \mathrm{C} \pm 5^{\circ} \mathrm{C}$. The billet was obtained by several forging/annealing steps in the $\beta$ (above $\beta$ transus) and $\alpha / \beta$ domain (at $760^{\circ} \mathrm{C}$ ). Samples were extracted from the core of the billet. A controlled microstructure composed of primary $\alpha_{\mathrm{p}}$ nodules embedded in a $\beta$ matrix was obtained by carrying out additional heat treatment of $760^{\circ} \mathrm{C}$ for 1 hour followed by water quenching on the samples. Moving forward, this is referred to as "as-forged" state. All investigated specimens had identical microstructure.

Several sections were cut and polished to observe the microstructure under the scanning electron microscope (SEM). The volume fraction of the primary $\alpha_{\mathrm{p}}$ phase was measured to be 15 $\pm 3 \%$. The $\alpha_{\mathrm{p}}$ nodules were mostly equiaxed with an average diameter of 2-3 $\mu \mathrm{m}$ from several backscattered images using ImageJ software [50]. As expected, no secondary $\alpha$ lamellae $\left(\alpha_{\mathrm{s}}\right)$ were present. Additional Electron Back-Scattered Diffraction (EBSD) measurements were carried out using a Zeiss AURIGA 40 electron microscope equipped with the AZTEC Oxford instrument system and the Symmetry camera. Fig. 1 shows the multi-scale microstructural aspects of the Ti-1023 as-forged alloy. Several orientation maps were acquired with $0.5 \mu \mathrm{m}$ step size (much smaller than the average $\alpha$ nodules diameter).

An EBSD analysis revealed that the microtexture consists of millimetric long prior $\beta$ grains along the forging axis (with an aspect ratio between $8-10$ ) as a result of the forging process. These long prior $\beta$ grains were partially fragmented in equiaxed sub-grains (with misorientation angles of $3^{\circ}-15^{\circ}$ ) of diameters ranging from
5 to $10 \mu \mathrm{m}$. The $\alpha_{\mathrm{p}}$ nodules were located at the prior $\beta$ grains and the $\beta$ sub-grain boundaries.

Neutron diffraction analysis was performed on a $1 \times 1 \times 1 \mathrm{~cm}^{3}$ sample to characterize the crystallographic texture of both $\alpha$ and $\beta$ phases at the 6T1 spectrometer of the French neutron source at LLB, using a wavelength of $1.159 \AA$. Complete pole figure measurements were carried out for three $\beta$ reflections $(110,200,311)$ and five $\alpha$ reflections (00.2, 11.0, 10.0, 10.1, 10.2). The Orientation Distribution Function (ODF) was calculated using the spherical harmonics method thanks to the ATEX software [51]. Measured pole figures revealed a rather moderate texture with a texture index of $\sim 2$ for the $\alpha$ phase and $\sim 3$ for the $\beta$ phase. Furthermore, most of the grains are preferably aligned along the $<110>\beta$ direction parallel to the forging axis of the billet, which is also the loading axis of the samples, see Fig. 2. Pole figure analysis from Fig. 2 indicates that the Burgers Orientation Relationship (BOR) between the $\alpha_{\mathrm{p}}$ and $\beta$ phase is still respected in average (all poles of $\{0001\}_{\alpha}$ and $\{110\}_{\beta}$ overlap as well as $<11 \overline{2} 0>_{\alpha}$ and $<111>_{\beta}$ ).

\subsection{In-situ high energy X-ray diffraction tensile experiments}

The in-situ high energy X-ray diffraction (HEXRD) tensile experiments were carried out at room temperature at the I12 beamline in the Diamond Light Source, UK [52]. Cylindrical specimens having a diameter of $1 \mathrm{~cm}$ were cut from the core of the billet using electrical discharge machining (EDM). These samples were then heat-treated to obtain the as-forged microstructure and were then machined into tensile specimens with gauge sections of $4 \mathrm{~mm}$ in diameter and $26 \mathrm{~mm}$ in length conforming with the ASTM-E8 standards [53].

Fig. 3 shows the complete experimental setup. A monochromatic beam of $70.45 \mathrm{keV}$ (wavelength $\lambda=0.17599 \AA$ ) with a crosssection of $500 \times 500 \mu \mathrm{m}^{2}$ was employed in transmission geometry, leading to a diffraction volume of $0.5 \times 0.5 \times 4 \mathrm{~mm}^{3}$. This diffraction volume corresponds to $6-8$ prior $\beta$ grains, however, it is reminded that the $\beta$ grains are fragmented into $\beta$ sub-grains of $5-10$ $\mu \mathrm{m}$ diameter thus providing enough grain statistics to form complete and continuous Debye-Scherrer diffraction rings, refer to 2D diffractogram in Fig. 3. The diffracted X-rays rings were recorded on a 2D Pilatus $2 \mathrm{M}$ CdTe area detector $(1475 \times 1679$ pixels, pixel size $172 \mu \mathrm{m}$ ) kept at $\sim 0.9 \mathrm{~m}$ from the specimen. Thanks to the high X-ray flux and fast readout time of the detector, the diffraction patterns were sampled continuously every $\sim 1$ s.
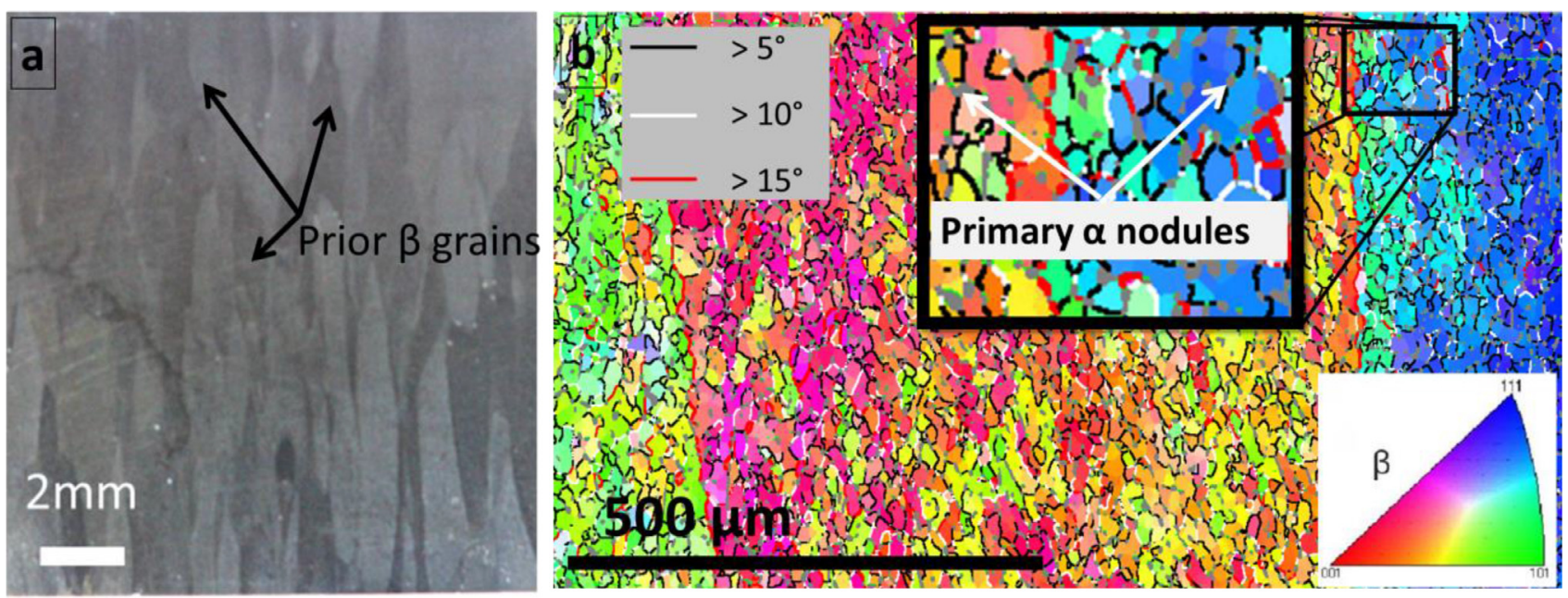

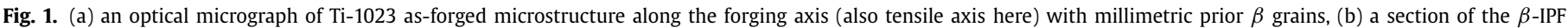

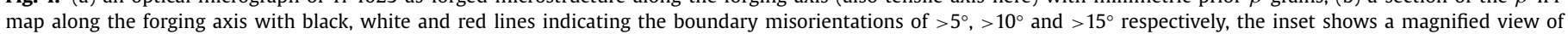
$\beta$-IPF map with primary $\alpha$ nodules in gray. 


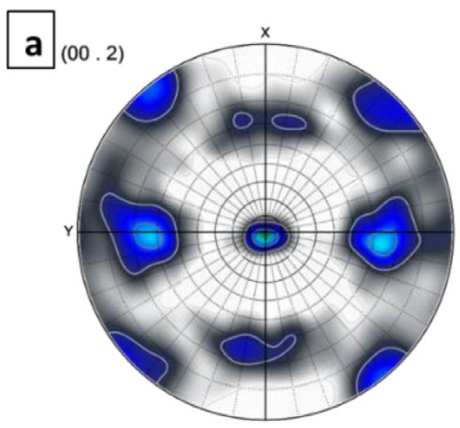

Min: 0.20, Max: 2.77

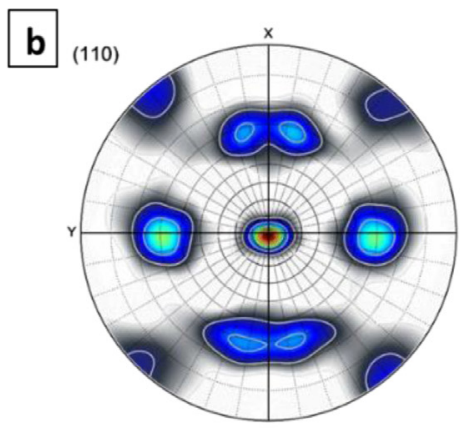

Min: 0.05, Max: 4.18

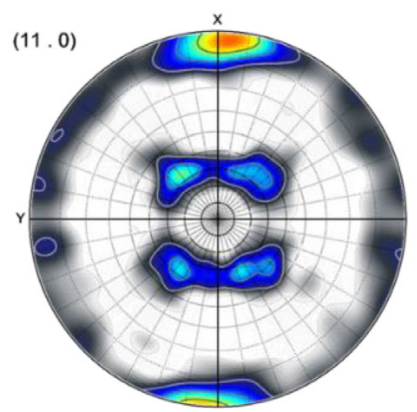

Min: 0.06, Max: 3.64

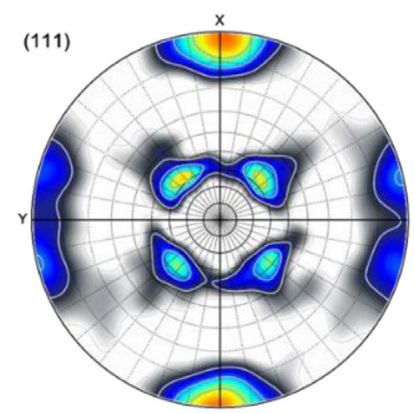

Min: 0.15, Max: 3.62

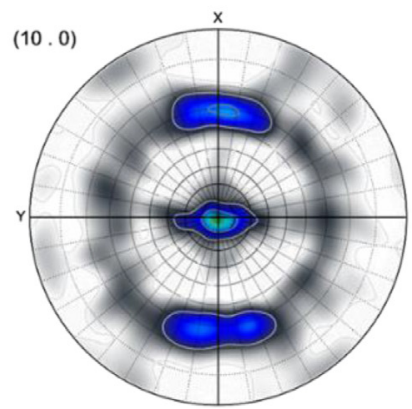

Min: 0.40, Max: 2.91

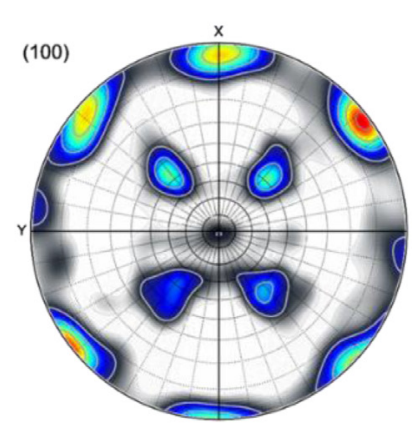

Min: 0.05, Max: 3.94

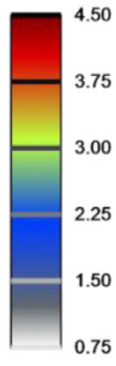

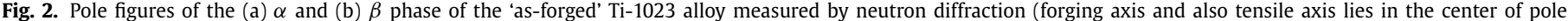
figures)

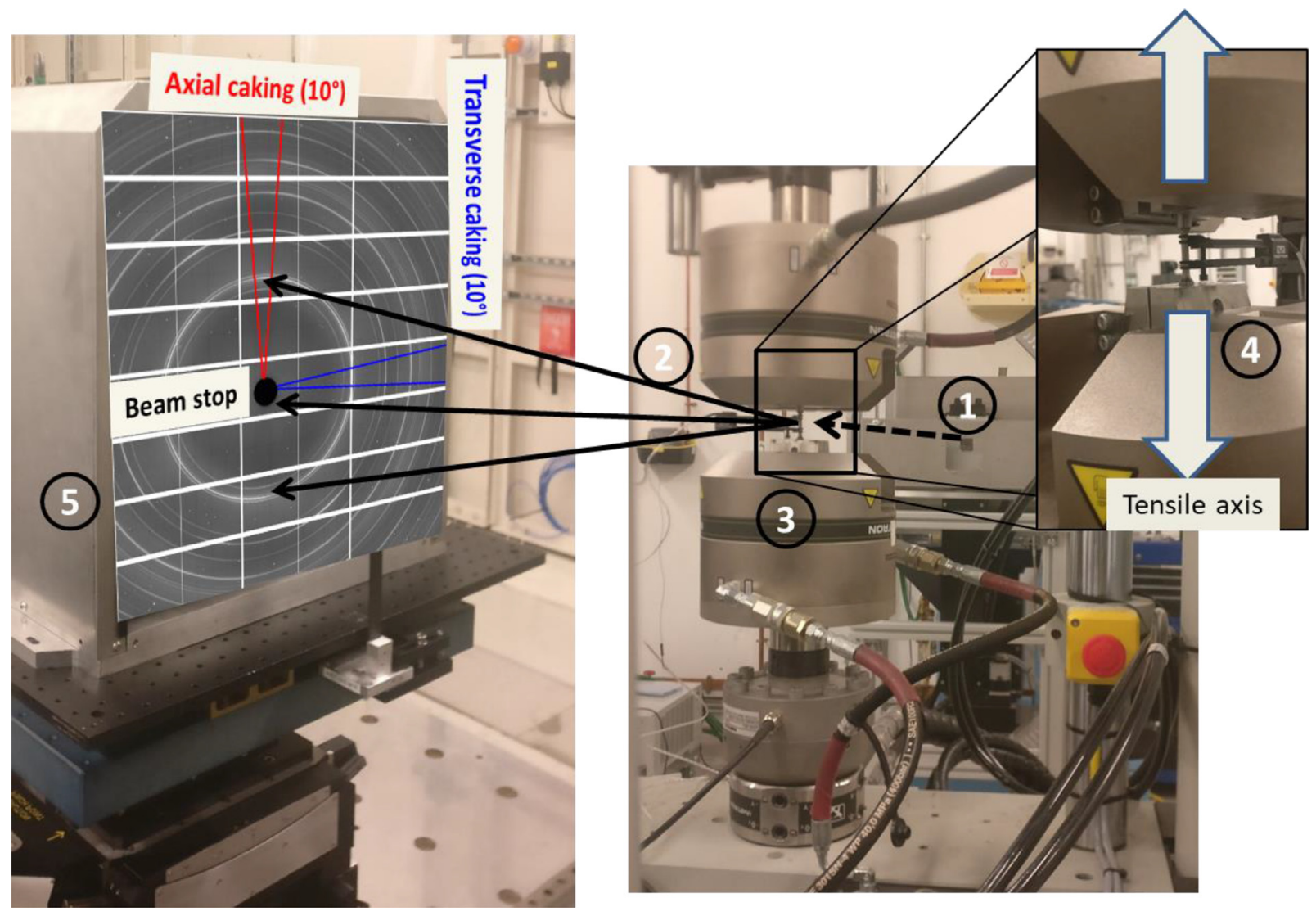

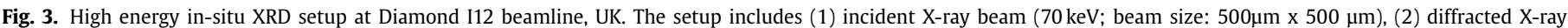

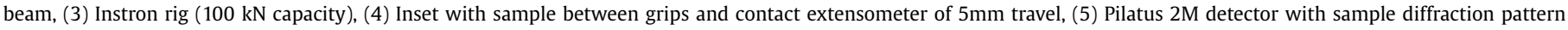
of the studied alloy $(1475 \times 1679$ : pixel size: $172 \mu \mathrm{m})$. 


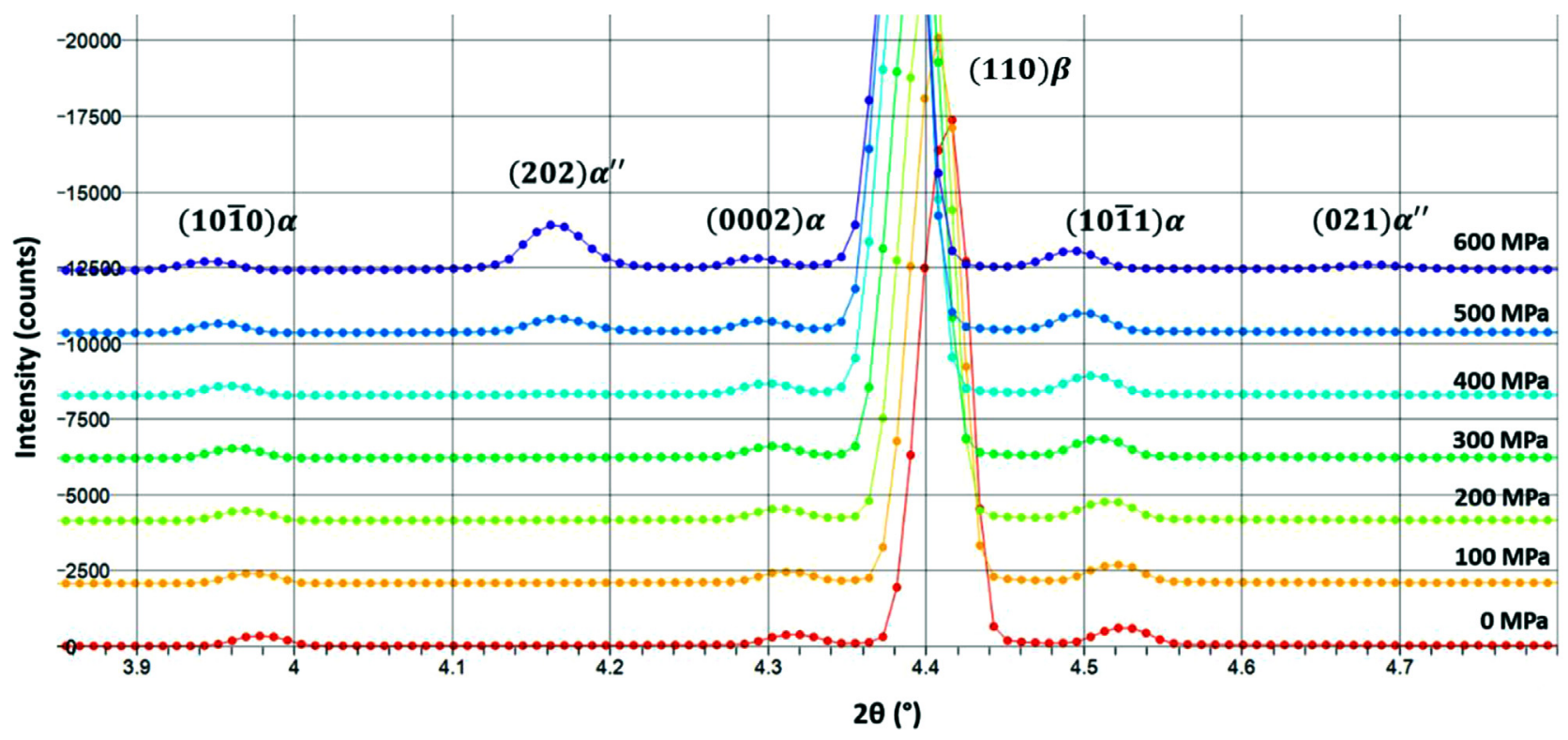

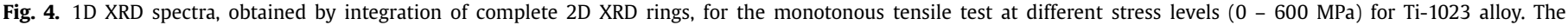
martensite peaks $\left(\boldsymbol{\alpha}^{\prime \prime}\right)$ appear close to $400 \mathrm{MPa}$

The deformation tests were performed on an Instron $100 \mathrm{kN}$ rig at a nominal strain rate of $10^{-4} \mathrm{~s}^{-1}$. The macroscopic strains were measured using a contact extensometer. The campaign includes a continuous tensile test, a load-unload cyclic test, and a series of increasing uni-axial tensile interrupted tests. This campaign was originally designed to study the elastic and plastic response of the material under various deformation paths and small plastic strain; only the elastic part is presented in this paper. The stressinterrupted tensile tests were performed to increase the diffracting volume i.e., every $50 \mathrm{MPa}$ increment up to $\sim 300 \mathrm{MPa}$ the sample was held at constant stress and a $72^{\circ}$ rotation of the Instron rig (along the tensile axis) was carried out with $2^{\circ} / \mathrm{s}$ and the diffraction patterns sampled every $2^{\circ}$ were then summed to get a single diffraction pattern. In Fig. 4, the stress-induced martensitic transformation peaks can be observed in the XRD spectra starting at $\sim 400 \mathrm{MPa}(\sim 0.6 \%$ total strain), which is not surprising considering the low volume fraction of the $\alpha$ phase [54-57]. Since our objective is to identify the SEC, data analysis was carried out in the purely elastic regime prior to phase transition (macroscopic stresses up to $\sim 300 \mathrm{MPa}$ ). Hence, the analysis is performed from the continuous tensile tests up to $300 \mathrm{MPa}$, four load-unload cycles performed at $(200,250,300,350 \mathrm{MPa})$, and stress-interrupted tensile tests up to $\sim 300 \mathrm{MPa}$. Rietveld refinement of XRD patterns at zero applied stress validates the SEM measurements of $15 \%$ volume fraction for the $\alpha$ phase.

XRD data analysis: The analysis of the XRD spectra provides important information concerning the deformation mechanisms and load transfer among different grains/ phases. For the current study, the XRD data analysis is limited to the lattice strain evolution with applied stress. However, considering the nature of the study, several aspects of XRD data processing that can result in additional uncertainties are carefully evaluated. The common source of uncertainty comes from the geometrical calibration of the experimental setup [58,59]. The errors are introduced possibly during the conventional three-step data analysis process: (i) geometrical calibration of the experimental setup, (ii) conversion of the 2D azimuthal data to $1 \mathrm{D}$ ( $2 \theta$ vs intensity) and (iii) peak-fitting for lattice strain evaluation. The diffraction data collected here exhibit a good quality with reduced background and noise levels (maximum peak intensity 18000 cts, signal/noise ratio 130).
The geometrical calibration of the detector was performed by a standard calibrant powder of $\mathrm{CeO}_{2}$ (SRM674 from NIST). The recorded 2D XRD rings are processed by "caking" [60] along the azimuth $\psi$. Both the axial and transverse direction (refer to Fig. 3) caking is carried out (here caking angle of $\Delta \psi= \pm 5^{\circ}$ is used). The integration along $\psi$ yields a 1D diffraction pattern. Additionally, the characterization of the image noise was done using multiple images acquired under the same geometrical configuration as in [61]; this indicates the photon noise source to be the sole contributor (not shown here) and therefore, an error model where the standard deviation of intensity is proportional to the square root of average intensity is employed during the peak fitting. The 1D pattern is then fitted with a Pseudo-Voigt function to resolve parameters related to the shape of the peak, the position $(2 \theta)$, and Full-Width Half Maximum (FWHM). Each peak/ reflection is treated separately during the fitting. In the Ti-1023 alloy with $\alpha+\beta$ phases, the majority of the $\alpha$ and $\beta$ diffraction peaks could be satisfactorily fitted, while the overlapping $\alpha / \beta$ peaks were ignored. The XRD data calibration and integration were carried out using the PyFAI library [62], while the peak fitting was carried out using Fityk software [63].

The in-situ XRD data interpretation is based on the relative changes in the measured values of the Bragg angle $\theta$. The lattice strain $\varepsilon_{h k l}$ is represented in microstrain $\left(10^{-6}\right)$ units by the following relation:

$\varepsilon_{h k l}=-\cot \left(\theta_{h k l}\right) \Delta \theta_{h k l} \times 10^{6}$

where $\Delta \theta_{h k l}=\theta_{h k l}-\theta_{0, h k l}$ is the difference in peak position calculated from the current $\theta_{h k l}$ and the $\theta_{0, h k l}$ calculated at the start of the test. Fig. 5 shows the XRD peak analysis for (1010) $\alpha$ reflection plotted with respect to applied stress. For better comparison and representation, both the lattice strains and applied stresses are normalized by dividing them with the magnitude of maximum applied stress of each respective test (around $300 \mathrm{MPa}$, which slightly varies between tests), such that the resulting normalized strains have unit microstrain/MPa. Fig. 5a shows the normalized macroscopic strain-stress curves with the dashed line representing the linear regression fit of the data from all tests. Fig. $5 \mathrm{~b}$ and $5 \mathrm{c}$ show the normalized axial and transverse lattice strains of $(10 \overline{10}) \alpha$ reflection along with the linear regression. Very similar data quality 

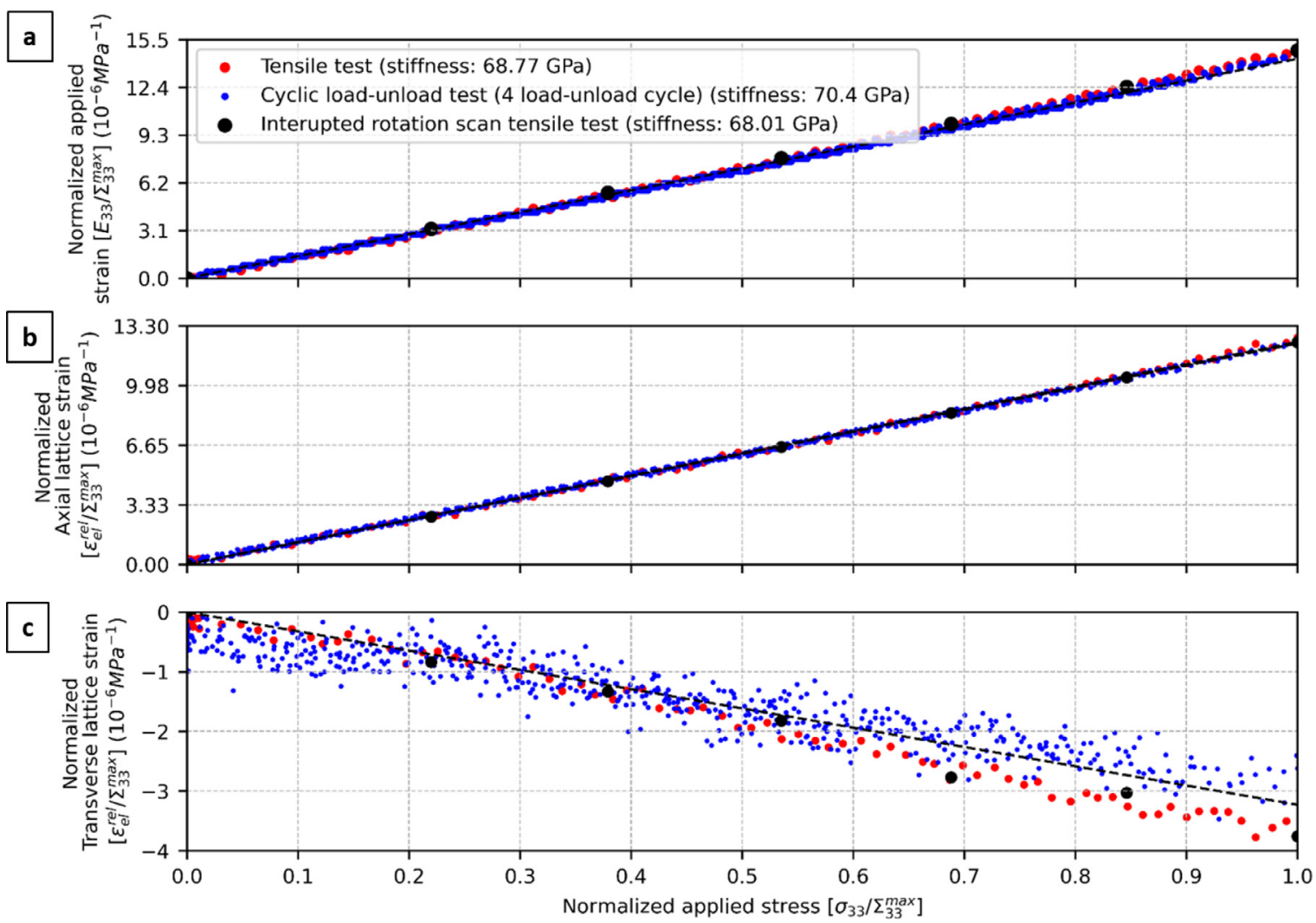

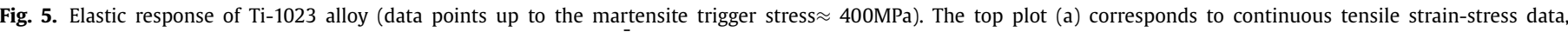

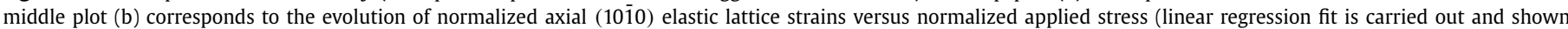

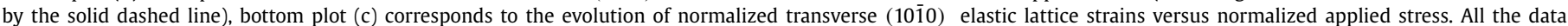
points are normalized by dividing them with maximum applied stress of the respective test (see text).
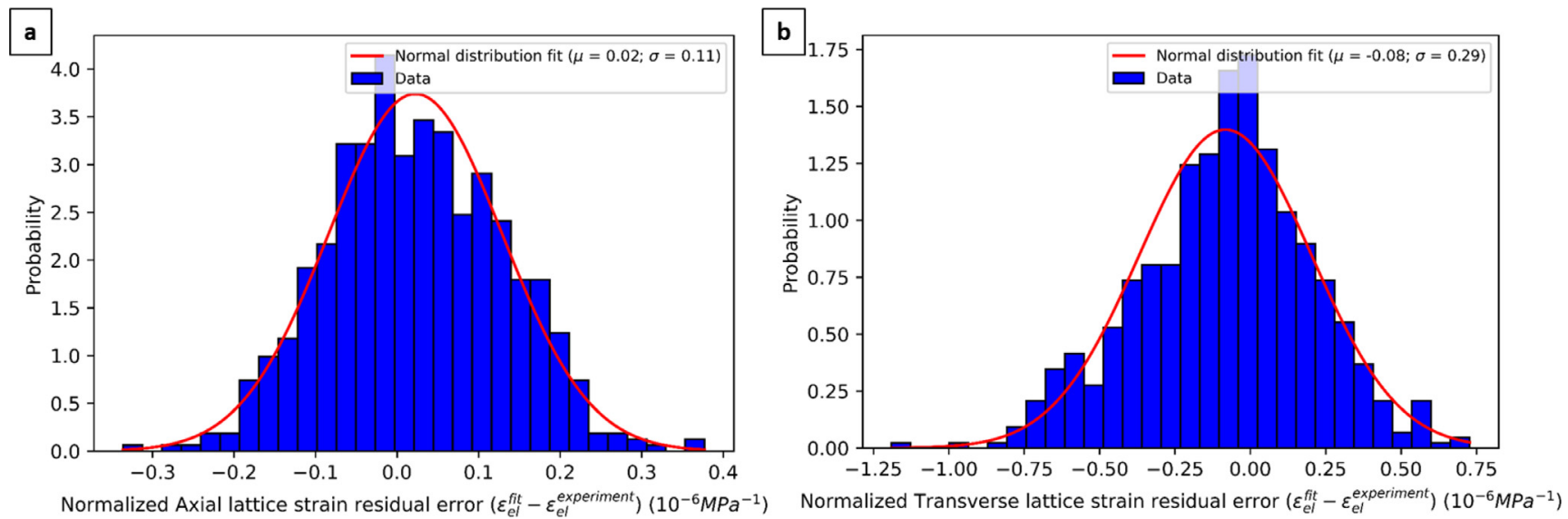

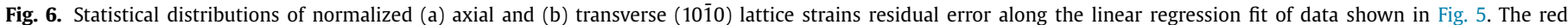
line is the associated fit of the dispersion by a normal distribution, the parameter of which is indicated in the legend.

has been obtained for other (hkl) reflections for both the $\alpha$ and $\beta$ phases.

The linear regression fit data is used as the average lattice strains and the scatter of experimental data at given stress around the fit is taken as the standard deviation for lattice strains. Fig. 6 shows the distribution of lattice strains from the different tests compared to their linear fit for both axial and transverse directions at applied unit stress. Following this procedure, other axial and transverse reflections of both $\alpha$ and $\beta$ phases are processed and are presented in the results section. All lattice strains for both $\alpha$ and $\beta$ phases are found to follow a nearly normal distribution similar to Fig. 6. It should also be noted that not all reflections could be used: especially, the stiffest (222) $\beta$ reflection is out of the detector capture range; the reflections (211) $\beta$ and (1013) $\alpha$ is not available due to peak-overlapping and the (0002) $\alpha$ transverse reflection lie in the detector dead zones.

It is reminded here that the observed lattice strain variability includes errors coming from data acquisition (e.g. photon noise), data processing and the variation of lattice strains from one experiment to another (as illustrated in Fig. 4c). The obtained dispersions in the lattice strains are representative of the material itself and provide a realistic range of average strains for the Bayesian inference to fit. Other relevant error sources during the experimental acquisition were found to be minimum/ negligible. In sum- 
mary, the experimental data used for the Bayesian inference include, along with their variability,

- 8 axial reflections and 4 transverse reflections for the $\alpha$ phase,

- 4 axial reflections and 2 transverse reflections for the $\beta$ phases, and

- the effective axial elastic stiffness of the material.

These data are indicated in Table 2 and plotted in Fig. 7.

\subsection{Bayesian framework with HEXRD and elastic self-consistent (ELSC) modeling}

Description of the elastic self-consistent (ELSC) model: The self-consistent scheme is one of the most popular homogenization methods to simulate the behavior of multiphase polycrystalline materials. This framework $[37,38]$ is implemented here to describe the elastic properties of the two-phase $(\alpha+\beta)$ polycrystalline aggregate. The ellipsoidal inhomogeneity (each representing a single crystal orientation in a given phase) is embedded in an infinite homogeneous equivalent medium (HEM), whose behavior is supposed to be the behavior of the multi-phase polycrystal. The constitutive relations for a heterogeneous linear elastic aggregate can be described as:

$E=M^{e f f}: \Sigma$

where the quantities $E$ and $\Sigma$ are the macroscopic strain and stress tensors and $M^{e f f}$ is the effective elastic compliance tensor of the polycrystal. The constitutive elastic behavior for each orientation ' $r$ ' between the average strains $\varepsilon^{r}$ and average stresses $\sigma^{r}$ over the volume occupied by $r$ yields:

$\varepsilon^{r}=m^{r}: \sigma^{r}$

where $m^{r}$ is the elastic compliance tensor of orientation $r$. In the homogenization theory, the stress concentration relationships between $\sigma^{r}$ and $\Sigma$ can be described as:

$\sigma^{r}=B^{r}: \Sigma$

where $B^{r}$ is the average stress concentration tensor for each orientation $r$ in a given phase $(\alpha / \beta)$. This tensor is evaluated considering each Eshelby's ellipsoidal inhomogeneity $r$ embedded in an infinite HEM subjected to homogeneous stress $\Sigma$ :

$B^{r}=\left(m^{r}+\tilde{M}\right)^{-1}:\left(M^{e f f}+\tilde{M}\right)$

where $\tilde{M}$ is the constraint tensor defined by the following expression [64]:

$\tilde{M}=\left(I-S^{E r}\right)^{-1}: S^{E r}: M^{e f f}$

where $I$ is the fourth-order unit tensor and $S^{E r}$ is the elastic Eshelby tensor [36] for a given inhomogeneity that can exhibit any ellipsoidal morphology. In equation (2), the effective elastic compliance tensor of the polycrystal $M^{e f f}$ is given by:

$M^{e f f}=\left\langle m^{r}: B^{r}\right\rangle:\left\langle B^{r}\right\rangle^{-1}$

where the brackets $\left\langle()^{r}\right\rangle$ denote the volume average over all the grains of the polycrystal: $\left\langle()^{r}\right\rangle=\sum_{r} f_{r}()^{r}$ with $f_{r}$ the volume fraction of orientation $r$. When replacing $B^{r}$ in equation (7) by its expression (5), one ends up with an implicit equation for $M^{\text {eff }}$ that is solved using a fixed-point algorithm [65]. For more details on the Eshelby tensor evaluation, the readers are referred to [64,66].

In the ELSC model, each mechanical phase is defined by its orientation (three Euler angles with the Bunge convention: $\phi_{1}, \Phi, \phi_{2}$ ) and a volume fraction $f_{r}$. For each grain orientation, if the plane normal of the given $(h k l)$ plane is aligned within $\pm 5^{\circ}$ of solid angle (same as experimental configuration, see Fig. 3 ) with the sample axis, then that grain contributes to that $(h k l)$ diffraction subset. To obtain the normalized elastic strains corresponding to different $(h k l)$ families of grains as in Fig. $5 \mathrm{~b}$ and $5 \mathrm{c}, \frac{\varepsilon_{h k l}^{\text {simulation }}}{\Sigma_{33}^{\max }}$, average strains (sometimes denoted as mesoscopic strains in the literature) of the diffracting grains are multiplied by their volume fraction and summed over the overall volume fraction of grains in the subset. This is then normalized by the maximum applied tensile stress. For a given loading direction of the specimen (uniaxial traction here), a compact representation of the $\varepsilon_{h k l}^{\text {simulation }}$ can be defined as the function of the diffraction condition (solid angle with the macroscopic axis $\left.\psi= \pm 5^{\circ}\right)$, texture $\left(\phi_{1}, \Phi, \phi_{2}\right)$, phase elastic constants, volume fraction $\left(\mathrm{f}_{\alpha}+\mathrm{f}_{\beta}=1\right)$, and morphology (spheres or prolates defined by the aspect ratio: $\alpha_{A R}, \beta_{A R}$ ).

$$
\begin{gathered}
\frac{\varepsilon_{\text {hkl }}^{\text {simulation }}}{\sum_{33}^{\text {applied }}}=f\left(\psi, \phi_{1}, \Phi, \phi_{2}, \mathrm{f}_{\beta}, C_{11}^{\beta}, C_{12}^{\beta}, C_{44}^{\beta}, C_{11}^{\alpha}, C_{12}^{\alpha}, C_{13}^{\alpha}, C_{33}^{\alpha},\right. \\
\left.C_{44}^{\alpha}, \alpha_{A R}, \beta_{A R}\right)
\end{gathered}
$$

It is worth noting that the elastic self-consistent model used here is very good in evaluating the effective elastic properties of polycrystalline materials, probably owing to the random character of the considered microstructure that qualitatively resembles the one of real polycrystals. Comparisons with full-field simulations that provide reference numerical solutions already showed that the ELSC scheme gives very accurate estimates for the elastic behaviour, not only for the effective behaviour but also for the first (grain average strain and stress) and second (fluctuation inside grain families) moments, eg. see [67-70]. One of the limitation of the ELSC is that advanced microstructural parameter, such as orientation relationship between neighboring grains, can hardly be taken into account.

Bayesian inference: Bayesian framework combined with standard linear model (SLM) [71], reduced-order FE models [45] has recently been used to estimate the intrinsic material properties of metals. Here the Bayesian inference technique is very briefly explained, for more details, the readers are referred e.g. to the work of Rappel et al. [71-74]. In brief, we are evaluating a simplified Bayes theorem [75]: considering a random variable " $\theta$ ", its probability density function for a given experimental dataset " $x$ " can be evaluated by applying Bayes theorem: the probability of " $\theta$ " occurring for a given set of " $\mathrm{x}$ ", or $P(\theta \mid x)$ (also called "posterior distribution") is expressed as statistical inference model below:

$P(\theta \mid x)=\frac{P(x \mid \theta) P(\theta)}{P(x)}$

where $P(\theta)$ is the probability distribution of the variable " $\theta$ " in the absence of " $\mathrm{x}$ " and is also called the "prior distribution". $P(x \mid \theta)$ is called the "likelihood function" that evaluates the probability of the data " $x$ " occurring for a given " $\theta$ ". The numerator of the Bayes formula is rather easy to formulate, while there are difficulties involved in calculating the denominator $P(x)$. In general, this quantity can be calculated by integrating over all possible values of " $\theta$ ",

$P(x)=\int_{\theta} P(x \mid \theta) d \theta$

For a simple and trivial model, this parameter is rather easy to evaluate and is often discarded as a normalization factor [45,71], while in some non-trivial models, this can be extremely difficult to estimate in a closed-form way. To simplify, this is often addressed by taking the ratio of two probabilities with Markov chain Monte Carlo (MCMC) [76]. In short, this constructs a Markov chain, 
which proposes a jump for the random variable " $\theta$ ", to carry out the Monte Carlo sampling. The jump proposed in a Markov chain is often carried out by a Metropolis sampler. The acceptance probability of a jump is calculated by the ratio of equation (9) for the current " $\theta$ current" and the proposed " $\theta$ proposed", thus cancelling out the denominator (equation 11). The proposed value is accepted if the acceptance is higher than a value randomly sampled between 0 and 1 :

Acceptance probability $=\min \left(1, \frac{P\left(x \mid \theta^{\text {proposed }}\right) P\left(\theta^{\text {proposed }}\right)}{P\left(x \mid \theta^{\text {current }}\right) P\left(\theta^{\text {current }}\right)}\right)$

The objective of Bayes formula (equation 9) is to calculate the posterior distribution, $P(\theta \mid x)$, such that the unknown material parameter " $\theta$ " can be deduced from sampling the posterior distribution. The integrals defining the Monte Carlo and Metropolis sampler algorithm can be further referred in [71].

Application to Ti-1023 alloy: For the identification of SEC of the $\beta$ phase in Ti-1023, a Bayesian framework is employed with the ELSC model to evaluate the likelihood function, which evaluates the probabilities of the macroscopic stiffness ( $\mathrm{YM}^{\text {simulation }}$ ) as well as the grain family elastic strains ( $\left.\varepsilon_{h k l}^{\text {simulation }}\right)$ against the experimental ( $\mathrm{YM}^{\text {Experiment }}$ ) stiffness and XRD lattice strains $\left(\varepsilon_{h k l}^{\text {Experiment }}\right)$ for different sets of material parameters. As can be seen from equation (8), the elastic strain $\varepsilon_{\text {hkl }}^{\text {simulation depends on } 15}$ different variables (for fixed boundary conditions). As a preliminary approach, out of the 15 variables all but the SEC of the $\beta$ phase is assigned a fixed value based on the experimental observations.

- The ODF calculated from neutron diffraction data has been used to discretize the texture of both phases using the ATEX software [51]. The discretization yields $\sim 2250$ orientations for the $\beta$ phase and $\sim 4500$ orientations for the $\alpha$ phase weighted by volume fraction.

- The volume fraction for the $\alpha$ phase is fixed, $\mathrm{f}_{\alpha}=0.15\left(\mathrm{f}_{\beta}=\right.$ 0.85 ), based on Rietveld analysis of XRD patterns and SEM image analysis.

- The diffraction solid angle for simulation $\left(\psi= \pm 5^{\circ}\right)$ is also fixed to comply with the experimental caking angle.

- As a preliminary approach, the $\alpha$ and $\beta$ grains morphology are reasonably assumed to be equiaxed and therefore are represented as spherical inhomogeneities in the ELSC model (i.e. $\alpha_{A R}=1, \beta_{A R}=1$ ). However, this will be later changed to prolate morphology (section IVb) to study its influence on the identification process; in that case, the grain shape considered in the model actually rather corresponds to the $\beta$ sub-grains than to the elongated prior $\beta$ grains.

- The SEC of the $\alpha$ phase $\left(C_{i j}^{\alpha}\right)$ are fixed due to a substantial agreement reported in the literature. Therefore, the SEC identified by Hearmon et al [77] are considered, based on a previous study performed on the same alloy [42]: $C_{11}^{\alpha}=160 \mathrm{GPa}, C_{12}^{\alpha}=90$ $\mathrm{GPa}, C_{44}^{\alpha}=46.5 \mathrm{GPa}, C_{33}^{\alpha}=181 \mathrm{GPa}, C_{13}^{\alpha}=66 \mathrm{GPa}$.

- The SEC of the $\beta$ phase $\left(C_{i j}^{\beta}\right)$ is kept free for optimization and inference.

The Bayes formula adapted to the Ti-1023 alloy reads

$P\left(D_{\text {simulation }} \mid D_{X R D}\right)=\frac{P\left(D_{X R D} \mid D_{\text {simulation }}\right) P\left(D_{\text {simulation }}\right)}{P\left(D_{X R D}\right)}$

where $D_{X R D}$ is a vector consisting of both micro and macro data: (hkl) dependent lattice strains $\left(\varepsilon_{h k l}^{\text {Experiment }}\right)$ and macroscopic stiffness $\left(\mathrm{YM}^{\text {Experiment }}\right)$. $D_{\text {simulation }}$ is an output vector obtained from the ELSC model consisting of (hkl) dependent elastic strains $\left(\varepsilon_{h k l}^{\text {simulation }}\right)$ and macroscopic stiffness ( $\left.\mathrm{YM}^{\text {simulation }}\right) . P\left(D_{\text {simulation }}\right)$ is the prior distribution and here it is defined as a uniform distribution, meaning that the values of the free variables are considered with equal probabilities within the material design space.

The likelihood function, $P\left(D_{X R D} \mid D_{\text {simulation }}\right)$, is evaluated from the difference between the experimental dataset and the output of the ELSC model. It is reminded again that the experimental data point in our case follows a Gaussian distribution (see Fig. 6). The probabilities of distribution in the Bayesian inference is often represented in the log scale for numerical stability and simplicity, instead of the usual $[0,1]$ interval. The log-likelihood function here is the sum of log-probabilities of the individual data point and can be written as,

$$
\begin{aligned}
& \log \left(P\left(D_{X R D} \mid D_{\text {simulation }}\right)\right) \propto \\
& -\sum_{i=1}^{n} W_{i}\left(\frac{1}{2} \log (2 \pi)+\log \left(S D_{i}\right)+\frac{\left(D_{\text {simulation }}^{i}-D_{X R D}^{i}\right)^{2}}{2 S D_{i}^{2}}\right)
\end{aligned}
$$

where ' $i$ ' represents data points (here ' $n$ ' $=19$, corresponding to the micro and macro experimental data points, see table 2). The $\mathrm{SD}_{\mathrm{i}}$ is the standard deviation and $W_{i}$ is the weight of each data point discussed in the next sections. The available data points sometimes may not produce a well-defined posterior distribution, i.e. the uncertainties of the distribution can be high. In such cases, additional experimental data points help to reduce the uncertainties or a careful weighing of the data points can lead to a welldefined posterior distribution. This is achieved through the $W_{i}$ term in equation 13. In the current study, the weighing is used as a way to counter-balance the fact that there is more $\alpha$ phase reflection, i.e., giving intrinsically less importance to the $\alpha$ phase data fitting since its volume fraction is much lower in reality.

We employ the adaptive Metropolis algorithm to search the material space during the sampling to increase efficiency. The Metropolis algorithm proposes a small increment of the available free variables based on their variance and a new probability density function is calculated. To avoid non-physical results in the simulations, only the SEC satisfying the Born elastic stability conditions [78] for cubic (equation 14 for $\beta$ phase) and transverse isotropy (equation 15 for $\alpha$ phase) are considered:

$C_{11}^{\beta}-C_{12}^{\beta}>0 ; \quad C_{11}^{\beta}+2 C_{12}^{\beta}>0 ; \quad C_{44}^{\beta}>0$

$C_{11}^{\alpha}>\left|C_{12}^{\alpha}\right| ; \quad 2\left(C_{13}^{\alpha}\right)^{2}<C_{33}^{\alpha}\left(C_{11}^{\alpha}+C_{12}^{\alpha}\right) ; \quad C_{44}^{\alpha}>0$

The efficiency of Metropolis sampling strongly depends on the initial guess and the variance of the proposal distribution of the free variables. For a good initial guess, a weighted least-squares optimization with an unconstrained Nelder-Mead algorithm was employed. MCMC sampling is also performed with additional free material parameters to assess the effect on the SEC identification and is discussed in the results section.

All the simulations are carried out using a script written in python interfacing pymc library for MCMC sampling [79] with Scipy's least-squares minimization [80] and a python implementation of the elastic self-consistent scheme developed at PIMM, Paris.

\section{Results}

\subsection{In-situ XRD lattice strain analysis}

The lattice (axial and transverse) strains for all considered peaks of both the $\alpha$ and $\beta$ phases at applied unit stress along with the macroscopic stiffness are presented in Table 2.

Both the axial and transverse lattice strains of all peaks present a Gaussian distribution. The standard deviations are the results of the variation of lattice strains from one experiment to another. Comparatively, the standard deviations of all reflections are quite 
Table 2

Statistics of axial and transverse lattice strains distribution for different reflections extracted from the regression fit for applied unit stress.

\begin{tabular}{|c|c|c|c|c|}
\hline$\alpha \mid \beta$ reflections & $\begin{array}{l}\text { Mean of axial lattice } \\
\text { strains distribution } \\
\left(10^{-6} \mathrm{MPa}^{-1}\right)\end{array}$ & $\begin{array}{l}\text { Standard deviation of axial } \\
\text { lattice strains distribution } \\
\left(10^{-6} \mathrm{MPa}^{-1}\right)\end{array}$ & $\begin{array}{l}\text { Mean of transverse lattice } \\
\text { strains distribution } \\
\left(10^{-6} \mathrm{MPa}^{-1}\right)\end{array}$ & $\begin{array}{l}\text { Standard deviation of } \\
\text { transverse lattice strains } \\
\text { distribution }\left(10^{-6} \mathrm{MPa}^{-1}\right)\end{array}$ \\
\hline $10-10 \alpha$ & 12.35 & \pm 0.11 & -3.58 & \pm 0.2 \\
\hline $0002 \alpha$ & 9.16 & \pm 0.14 & - & - \\
\hline $10-11 \alpha$ & 11.46 & \pm 0.2 & -3.88 & \pm 0.2 \\
\hline $11-20 \alpha$ & 10.70 & \pm 0.1 & -4.81 & \pm 0.18 \\
\hline $20-20 \alpha$ & 12.22 & \pm 0.1 & -3.73 & \pm 0.24 \\
\hline $0004 \alpha$ & 9.53 & \pm 0.18 & - & - \\
\hline $20-22 \alpha$ & 12.00 & \pm 0.18 & - & - \\
\hline $20-23 \alpha$ & 11.38 & \pm 0.2 & - & - \\
\hline $110 \beta$ & 13.70 & \pm 0.15 & - & - \\
\hline $200 \beta$ & 15.87 & \pm 0.1 & -7.23 & \pm 0.3 \\
\hline $220 \beta$ & 13.09 & \pm 0.2 & -5.02 & \pm 0.25 \\
\hline $310 \beta$ & 15.04 & \pm 0.1 & - & - \\
\hline Young's modulus (YM) & 69.77 & \pm 0.8 & - & - \\
\hline
\end{tabular}

a
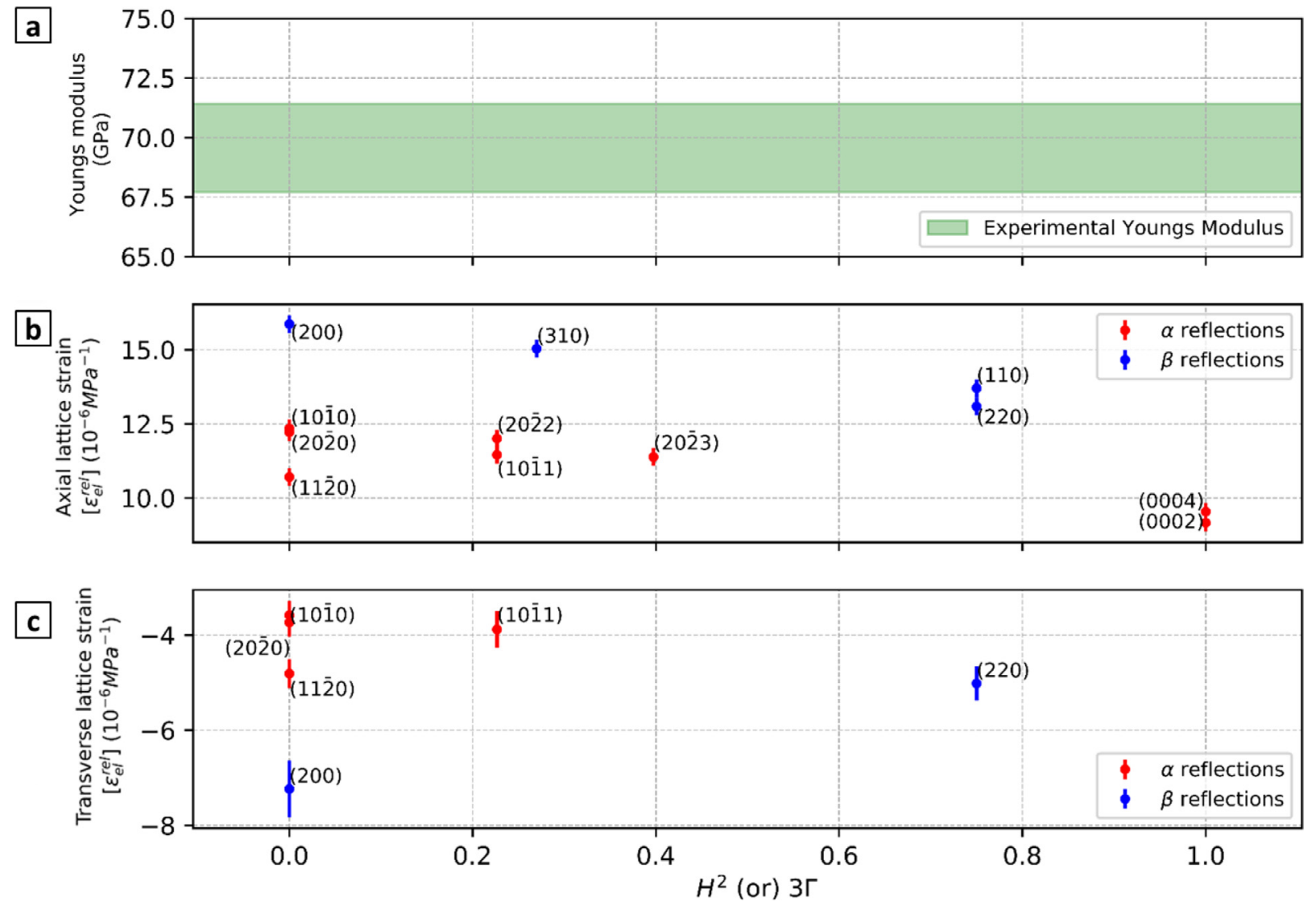

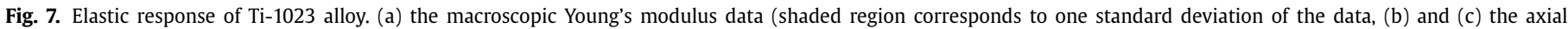

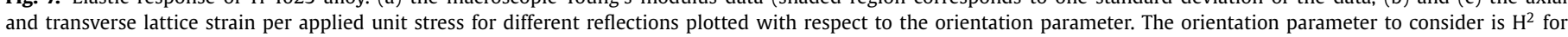
the $\mathrm{HCP} \alpha$ phase and $3 \Gamma$ for the $\mathrm{BCC} \beta$ phase.

low, suggesting very minimal variation in texture and microstructure among the tested specimens, and good XRD data.

To better understand the relationship between the different (hkl) peaks, the lattice strains are plotted as a function of the orientation parameter [81]. The orientation parameter effectively expresses the orientation dependency of the elastic stiffness in terms of the Miller indices. The orientation parameter $\Gamma^{\mathrm{hkl}}$ for the cubic system varies between 0 and $1 / 3$ i.e. 0 for the most compliant reflection (hkl) and $1 / 3$ for the stiffest (hkl) and is defined as:

$\Gamma^{\mathrm{hkl}}=\frac{h^{2} k^{2}+h^{2} l^{2}+k^{2} l^{2}}{\left(h^{2}+k^{2}+l^{2}\right)^{2}}$ while for the hexagonal system (in hkl notation), the orientation parameter varies from 0 (most compliant (hk.l)) to 1 (stiffest (hk.l)) and is defined as:

$H^{2}=\frac{l^{2}}{\frac{4}{3}\left(\frac{c}{a}\right)^{2}\left(h^{2}+k^{2}+h k\right)+l^{2}}$

where h, k, 1 are the Miller indices of the considered reflection and (c, a) are the unit cell dimensions of the hexagonal crystal system.

Fig. 7a presents the range of macroscopic stiffness (Young's modulus) for the as-forged Ti-1023 alloy. Fig. 7b and 7c present the grain-scale lattice strains (axial and transverse) at applied unit stress as a function of the orientation parameter. The stiffest and compliant (hkl) reflections can be identified directly from the plot 

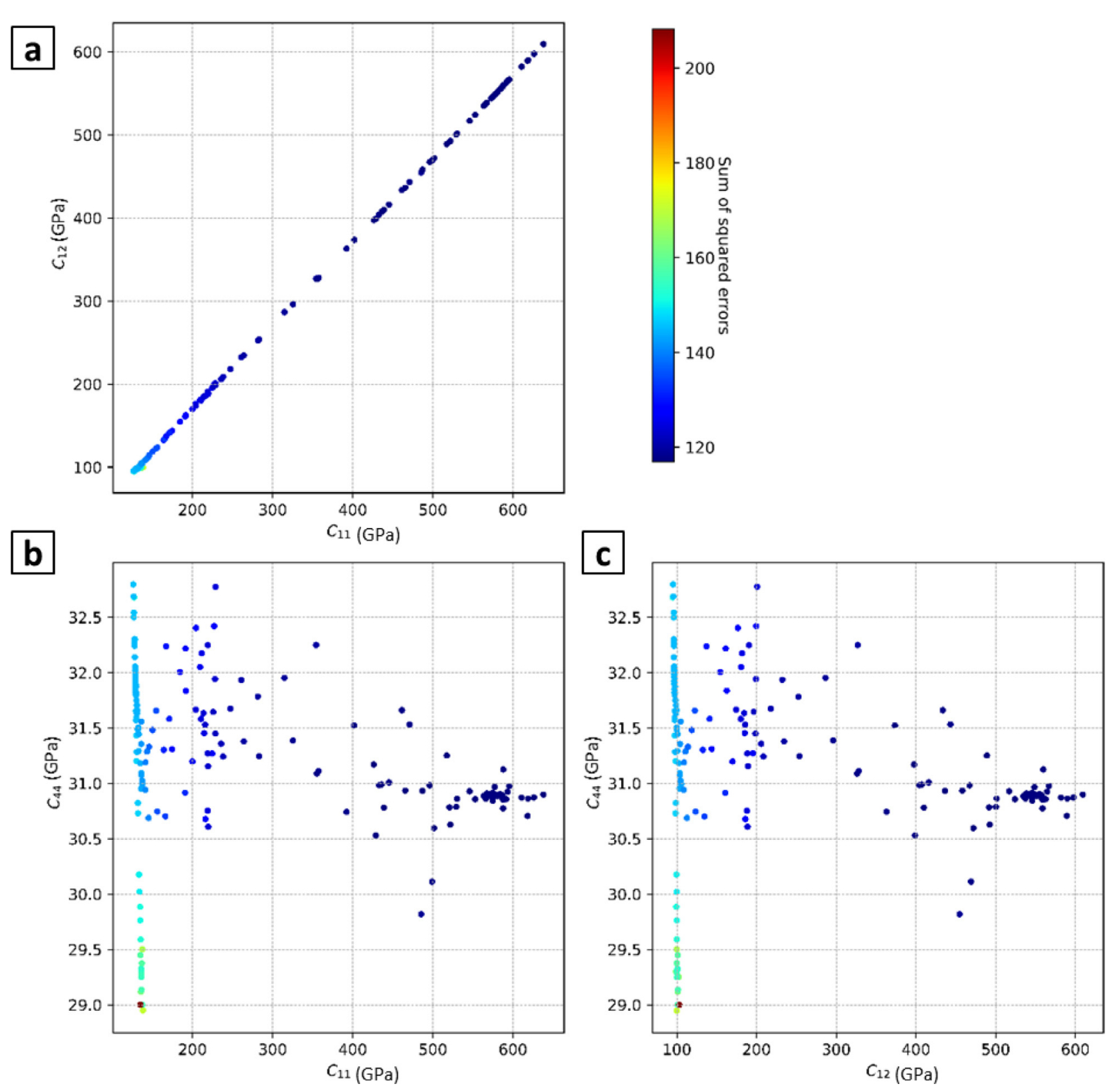

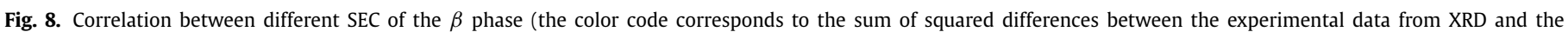
simulated data from the self-consistent model).

thanks to the orientation parameter. In the axial direction for the $\beta$ phase, the most complaint is the (200) grain family. For the $\alpha$ phase, the most compliant is the (1010) reflection and the stiffest is the (0002) reflection. While in the transverse direction (200) is still the most compliant one for the $\beta$ phase and (1120) is the most compliant one for the $\alpha$ phase. By transverse isotropy the planes perpendicular to the (0002) basal plane in $\alpha$ phase i.e. the (1010) and $(1 \overline{2} 0)$ should have similar elastic properties. The difference between the behavior of $(10 \overline{1} 0)$ and $(11 \overline{2} 0)$ probably arises due to the constraints in the local environment of the grains, as the environment of $\alpha$ grains is not random but associated with a phase transition. This has also been observed in near $\alpha$ Ti-834 [33]. This experimental data set ( 1 data point for Young's modulus, 6 data point for the $\beta$ phase, and 12 data point for the $\alpha$ phase) along with its variance is used to evaluate the SEC of the $\beta$ phase with the Bayesian model.

\subsection{Elastic constant identification from in-situ HEXRD and Bayesian inference}

In this section, the results from Bayesian inference concerning the assessment of the range of single-crystal elastic constants, for the cubic $\beta$ phase in Ti-1023, conforming to the experimental XRD dataset are presented.

Firstly, a least-squares optimization with the ELSC model was carried out to find a good initial guess for the three SEC of the $\beta$ phase $\left(C_{11}^{\beta}, C_{12}^{\beta}, C_{44}^{\beta}\right)$ that describes the experimental data well ( $\varepsilon_{\text {hkl }}^{\text {Experiment }}$ and macroscopic stiffness). Fig. 8 shows the obtained correlation among the SEC of the $\beta$ phase for which the sum of squared errors is less than 200 . The least-squares minimization indicates that the two constants $C_{11}^{\beta}$ and $C_{12}^{\beta}$ shares a linear relationship i.e., $C_{11}^{\beta} \approx C_{12}^{\beta}+33$ GPa. The $C_{44}^{\beta}$ identified by the least-squares 
Table 3

Least-squares analysis of $C_{i j}^{\beta}$ parameter.

\begin{tabular}{llllll}
\hline & $C_{11}^{\beta}(\mathrm{GPa})$ & $C_{12}^{\beta}(\mathrm{GPa})$ & $C_{44}^{\beta}(\mathrm{GPa})$ & $\mathrm{A}$ & $\mu^{\prime}(\mathrm{GPa})$ \\
\hline This study (weighted Least-squares fit) & 136 & 103 & 29 & 1.76 & 16.5 \\
\hline
\end{tabular}

( $31 \mathrm{GPa}$, refer to Fig. $8 \mathrm{~b}$ and $8 \mathrm{c}$ ) is comparatively lower and closer to the low modulus $\beta$ Ti-alloys. Adding both micro and macro experimental parameters to the minimization problem has narrowed down the exploration range of $C_{i j}^{\beta}$ constants. However, the leastsquares analysis failed to give a unique minimum value, instead multiple $C_{11}^{\beta}, C_{12}^{\beta}$ values satisfy both experimental lattice strains and macroscopic stiffness, refer to similar and multiple low sumof-squared errors in Fig. 8. This implies that the considered experimental data of lattice strains and macroscopic stiffness are more sensitive to the cubic shear moduli $\mu^{\prime \prime}=C_{44}^{\beta}$ and $\mu^{\prime}=\frac{C_{11}^{\beta}-C_{12}^{\beta}}{2}$, than to the bulk modulus of the $\beta$ phase, $K=\frac{\left(C_{11}^{\beta}+2 C_{12}^{\beta}\right)}{3}$. This is common for the $\beta$ phase in Ti-alloys. Studies on the Ti-24Nb-4Zr8Sn [18] and Ti-36Nb-5Zr [17] alloys have shown similar sensitivity of the XRD data to the SEC of the $\beta$ phase. This is often addressed by fixing the value of $C_{11}^{\beta}$ and $C_{12}^{\beta}$ (or bulk modulus $\mathrm{K}$ ) constants.

The absence of a unique value found by the least-squares minimization, suggests that the associated uncertainties for the $C_{11}^{\beta}, C_{12}^{\beta}$ are much higher than for $C_{44}^{\beta}$. Instead of fixing the bulk modulus $\mathrm{K}$, as a first approach, weights $\mathrm{W}_{\mathrm{i}}$ equal to the volume fraction of their corresponding phases were assigned to each data points i.e. more importance is given to lattice strains of the significant phase. Here we have considered $W_{i}=0.15$ for all the 12 data points of the $\alpha$ phase, $\mathrm{W}_{i}=0.85$ for all the 6 data points of the $\beta$ phase, and $W_{i}=1.0$ for the macroscopic Young's modulus. It is reminded that this kind of rational approach of adding weight to the data point is critical in our case, as not many (hkl) reflections are available for building a complete dataset.

Least-squares optimization is carried out again with weights for each data point. Adding weights to the data point results in a local minimum being identified for the $C_{i j}^{\beta}$ that satisfy the experimental data set. The two constants $C_{11}^{\beta}$ and $C_{12}^{\beta}$ still shares a linear relationship i.e., $C_{11}^{\beta}-C_{12}^{\beta}=33 \mathrm{GPa}$. The $C_{i j}^{\beta}$ parameter with the lowest sum-of-squared error is presented in Table 3.

To measure the uncertainties associated with the identified $C_{i j}^{\beta}$ and the influence of different material parameters, MCMC simulations are carried out. The $C_{i j}^{\beta}$ values suggested by the least-squares optimization is used as an initial guess for the MCMC sampling. Similar weights to that of the least-squares are employed for the MCMC sampling. The crystallographic texture and the grain morphology both play an important role in the global and local elastic properties. Due to the analytical nature of the calculations, statistically significant orientation is fixed and is discretized from the ODF, while the grain morphology is varied. Hence two different cases (corresponding to grain as a sphere and a prolate spheroid) of MCMC sampling are carried out and the results are discussed below.

\section{Case I: Spherical grains}

MCMC simulations were carried out with priors having a uniform distribution of a given range, refer to Table 4. A large range of values for $C_{11}^{\beta}$ and $C_{12}^{\beta}$ was employed based on the inference from the least-squares results that suggests multiple values for $C_{i j}^{\beta}$.

A total of 250,000 successful iterations (with 150,000 iterations to tune the variance) were carried out to assess the range of SEC
Table 4

The available search range of values for the prior variables.

\begin{tabular}{ll}
\hline Variable & Search range $(\mathrm{GPa})$ \\
\hline$C_{11}^{\beta}$ & $70-500$ \\
$C_{12}^{\beta}$ & $60-500$ \\
$C_{44}^{\beta}$ & $25-60$ \\
\hline
\end{tabular}

Table 5

Correlation coefficient of the multivariate distribution presented in Fig. 9.

\begin{tabular}{llll}
\hline & $\boldsymbol{C}_{11}^{\beta}$ & $\boldsymbol{C}_{12}^{\beta}$ & $\boldsymbol{C}_{44}^{\beta}$ \\
\hline$C_{11}^{\beta}$ & 1 & 0.998 & -0.432 \\
$C_{12}^{\beta}$ & 0.998 & 1 & -0.376 \\
$C_{44}^{\beta}$ & -0.432 & -0.376 & 1 \\
\hline
\end{tabular}

possible to describe the experimental dataset. Fig. 9 shows the 2D marginal posterior distribution of the range of three SEC of the $\beta$ phase. The maximum a-posteriori estimate (MAP) of the multivariate distribution is close to the one suggested by the weighted least-squares estimate. The posterior distributions of $C_{i j}^{\beta}$ present a normal distribution with well-defined peaks.

The peak probability values of both $C_{11}^{\beta}, C_{12}^{\beta}$ and $C_{44}^{\beta}$ are within the literature identified values for the $\beta$ phase. The standard deviation on both $C_{11}^{\beta}$ and $C_{12}^{\beta}$ is rather large $(\sim 24 \mathrm{GPa})$ compared to that of the $C_{44}^{\beta}(\sim 1 \mathrm{GPa})$. The correlation coefficients of the multivariate distribution $\left(\mathrm{C}_{11}^{\beta}, \mathrm{C}_{12}^{\beta}\right.$ and $\left.\mathrm{C}_{44}^{\beta}\right)$ are presented in Table 5. A strong positive correlation can be seen between $C_{11}^{\beta}$ and $C_{12}^{\beta}$.

Fig. 10 shows the multivariate distribution of the anisotropy ratio (A), shear modulus $\left(\mu^{\prime}\right)$, and bulk modulus (K). The MCMCidentified anisotropy ratio $(\sim 1.7)$ lies in the lower spectrum of the literature range (1.4 to 8 ), refer to Fig. 10a. Fig. 10b presents a sharp peak for the bulk modulus with a MAP of $124 \mathrm{GPa}$.

The correlation coefficients of the multivariate distribution of anisotropy ratio, bulk modulus, and shear modulus are presented in Table 6. A strong negative correlation can be seen between the anisotropy ratio and the shear modulus.

Fig. 11 shows the agreement between the simulated and experimental dataset for the identified $C_{i j}^{\beta}$ constants. A reasonably good agreement is seen for both the $\alpha$ and $\beta$ phase lattice strains and also the Young's modulus lies within the experimental range, refer to the dashed lines for the median value of the distribution in Fig. 11. For the $\beta$ phase, good agreement is seen for the axial lattice strains, while the simulated transverse elastic strains are slightly different from the experimental data, especially the (220) reflection.

The SEC obtained by the Bayesian approach for the $\beta$ phase $\left(C_{11}^{\beta}=134.3 \pm 24.3 \mathrm{GPa}, \quad C_{12}^{\beta}=101.5 \pm 24.5 \mathrm{GPa}, \quad C_{44}^{\beta}=\right.$ $28.8 \pm 1.13 \mathrm{GPa}$ ) agrees well with the elastic behavior of the Ti1023 alloy i.e. both the micro and macro experimental data are fitted reasonably well with the ELSC model.

\section{Case II: Ellipsoidal grains (prolate spheroids)}

In this case, the effect of $\beta$ phase morphology is also quantified along with the SEC. The effect of morphology is often simplified and is rarely considered in the literature. Here, we have considered 


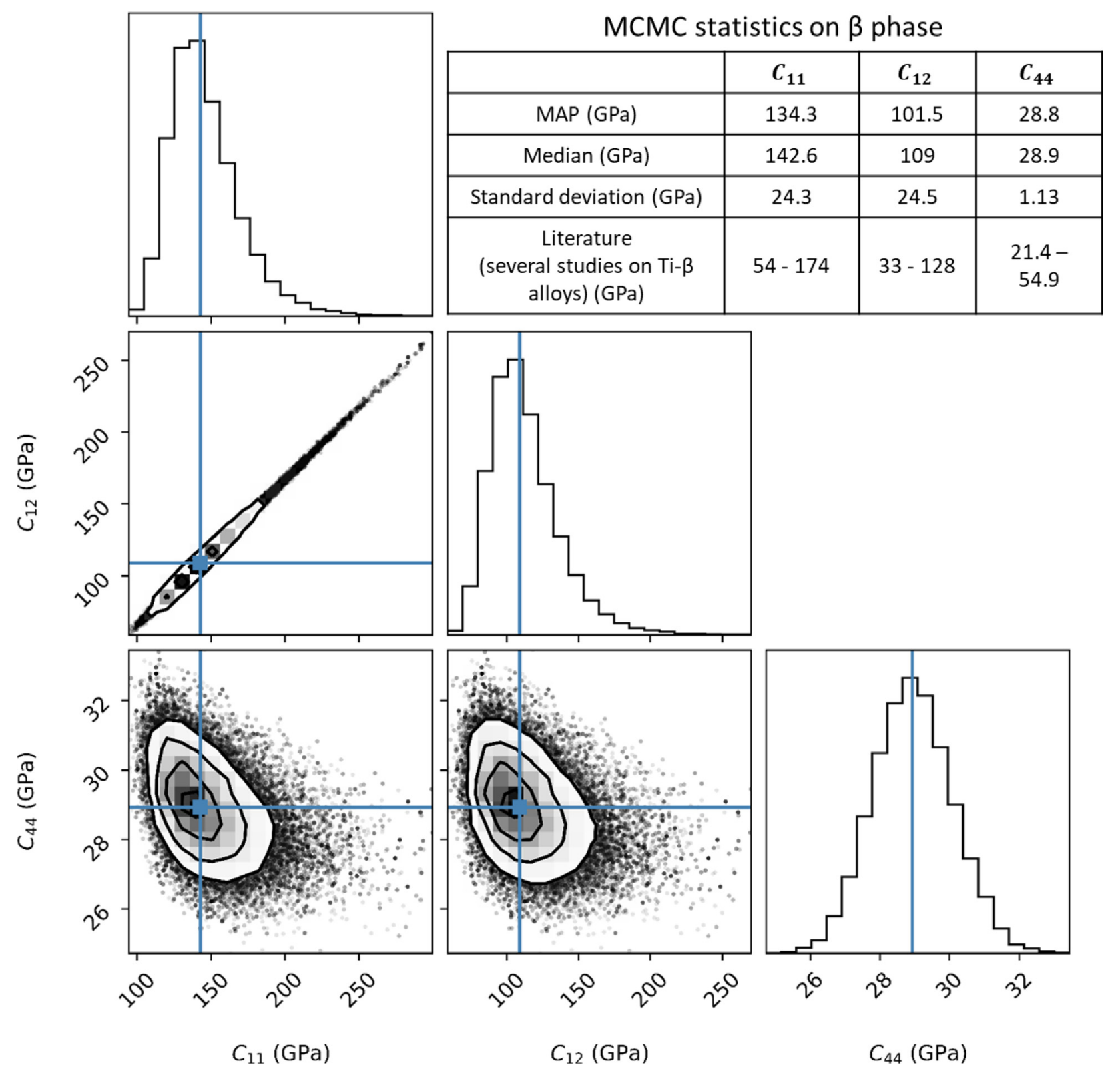

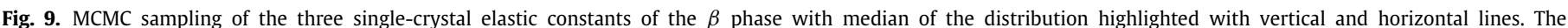

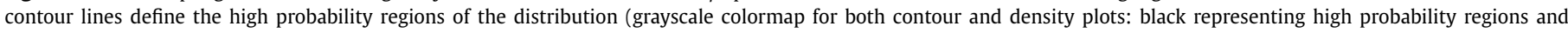
gray representing lower probability region).

Table 6

Correlation coefficient of the multivariate distribution presented in Fig. 10.

\begin{tabular}{llll}
\hline & Anisotropy ratio $(\mathrm{A})$ & Bulk modulus $(\mathrm{K})$ & Shear modulus $\left(\mu^{\prime}\right)$ \\
\hline Anisotropy ratio $(\mathrm{A})$ & 1 & -0.105 & -0.955 \\
Bulk modulus $(\mathrm{K})$ & -0.105 & 1 & -0.136 \\
Shear modulus $\left(\mu^{\prime}\right)$ & -0.955 & -0.136 & 1 \\
\hline
\end{tabular}

the morphology of the $\beta$ phase $\left(\beta_{A R}\right)$ to be a free variable in the MCMC analysis. This choice is motivated by the complex morphology of the $\beta$ grains revealed by EBSD analysis: prior $\beta$ grains are elongated (aspect ratio 8-10) and partially fragmented into subgrains locally disoriented up to $15^{\circ}$. Clusters of low angle misoriented sub-grains can act as one grain [82]. The morphology of the $\alpha$ phase remains fixed at $\alpha_{A R}=1$, this is motivated by image analysis clearly showing the globular shape. A total of 250,000 MCMC iterations (with 150,000 iterations to tune the variance) were car- ried out with priors having uniform distribution similar to Table 4. Additionally, the aspect ratio was allowed with a uniform distribution between $1.0<\beta_{A R}<10$. The morphology of the $\beta$ phase is considered to be of a prolate spheroid $(\mathrm{a}=\mathrm{b}<\mathrm{c})$ with the long axis of the ellipsoid aligned with the tensile axis (Fig. 1).

The 2D marginal posterior distributions of the $C_{i j}^{\beta}$ and $\beta_{A R}$ are shown in Fig. 12. Significant changes are seen in the posterior distribution of $C_{i j}^{\beta}$, with an aspect ratio $\left(\beta_{A R}\right)$ showing a peak value of 

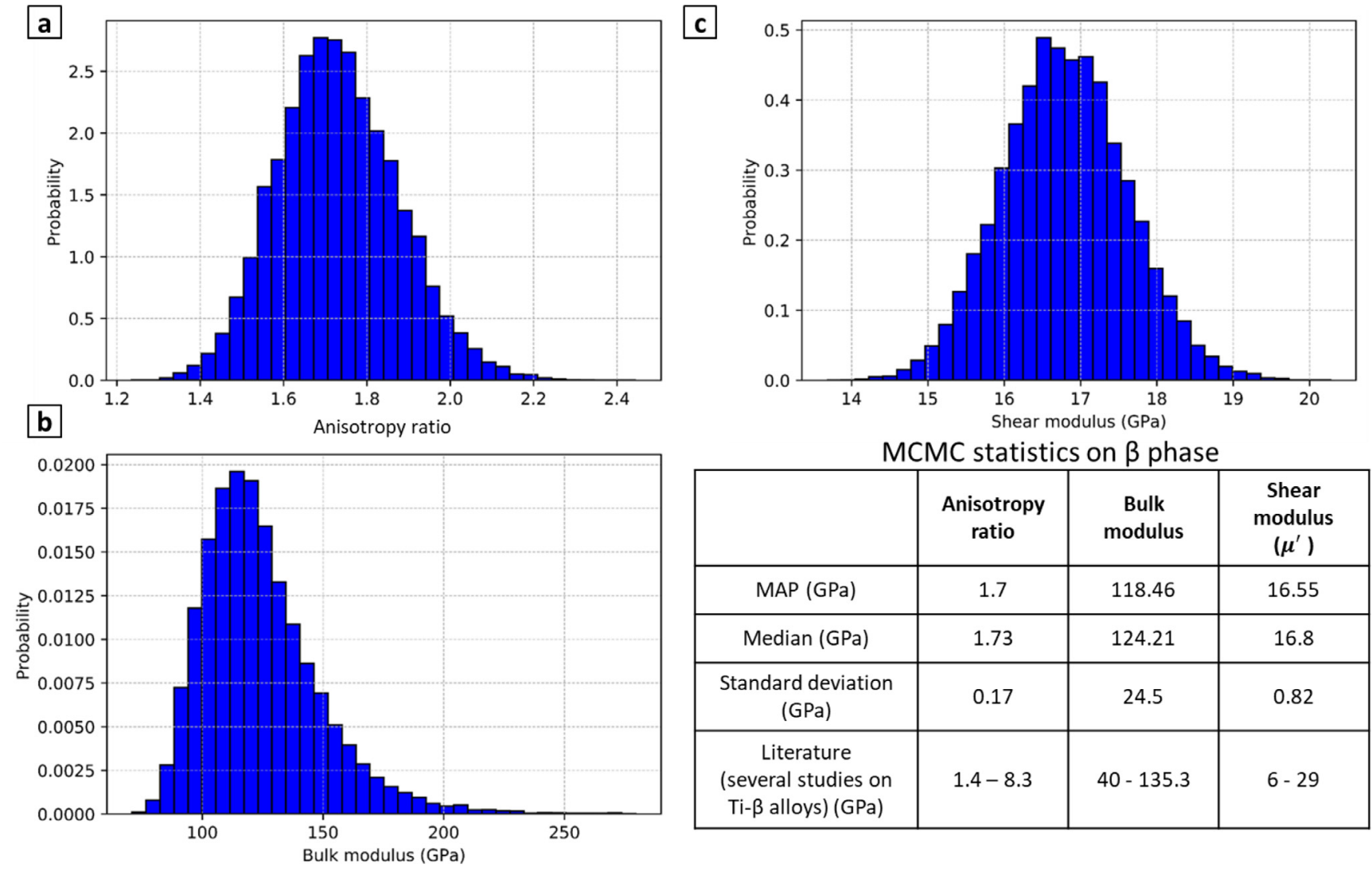

MCMC statistics on $\beta$ phase

\begin{tabular}{|c|c|c|c|}
\hline & $\begin{array}{c}\text { Anisotropy } \\
\text { ratio }\end{array}$ & $\begin{array}{c}\text { Bulk } \\
\text { modulus }\end{array}$ & $\begin{array}{c}\text { Shear } \\
\text { modulus } \\
\left(\boldsymbol{\mu}^{\prime}\right)\end{array}$ \\
\hline MAP (GPa) & 1.7 & 118.46 & 16.55 \\
\hline Median (GPa) & 1.73 & 124.21 & 16.8 \\
\hline $\begin{array}{c}\text { Standard deviation } \\
\text { (GPa) }\end{array}$ & 0.17 & 24.5 & 0.82 \\
\hline $\begin{array}{c}\text { Literature } \\
\text { (several studies on } \\
\text { Ti- } \beta \text { alloys) (GPa) }\end{array}$ & $1.4-8.3$ & $40-135.3$ & $6-29$ \\
\hline
\end{tabular}

Fig. 10. MCMC sampling of the anisotropy ratio (A), bulk (K) and shear ( $\left.\mu^{\prime}\right)$ moduli of the $\beta$ phase

Table 7

Correlation coefficients of the multivariate distribution presented in Fig. 12.

\begin{tabular}{lllll}
\hline & $C_{11}^{\beta}$ & $C_{12}^{\beta}$ & $C_{44}^{\beta}$ & Aspect ratio \\
\hline$C_{11}^{\beta}$ & 1 & 0.953 & -0.702 & -0.376 \\
$C_{12}^{\beta}$ & 0.953 & 1 & -0.462 & -0.177 \\
$C_{44}^{\beta}$ & -0.702 & -0.462 & 1 & 0.619 \\
Aspect ratio & -0.376 & -0.177 & 0.619 & 1 \\
\hline
\end{tabular}

3.8. The aspect ratio is lower than the experimental average of the prior $\beta$ grains and is consistent considering the experimental microstructure in which the prior $\beta$ grains are partially fragmented. The peak values of the posterior distribution of $C_{11}^{\beta}, C_{12}^{\beta}$ and $C_{44}^{\beta}$ are well within the literature range. In comparison to the previous case with spherical inclusion, the value of $C_{44}^{\beta}$ has increased while the shear and bulk modulus values have decreased significantly. Also, the standard deviation of $C_{11}^{\beta}, C_{12}^{\beta}$ constants have decreased, while it has increased for the $C_{44}^{\beta}$. The SEC obtained by the Bayesian inference is $\left(C_{11}^{\beta}=92.6 \pm 19.1 \mathrm{GPa}, \quad C_{12}^{\beta}=82.5 \pm 16.3 \mathrm{GPa}, \quad C_{44}^{\beta}=\right.$ $43.5 \pm 7.1 \mathrm{GPa})$. The correlation coefficients of the multivariate distribution $\left(C_{11}^{\beta}, C_{12}^{\beta}, C_{44}^{\beta}\right.$ and aspect ratio) are presented in Table 7. Aspect ratio has moderate positive correlation with $C_{44}^{\beta}$. The correlation between $C_{11}^{\beta}$ and $C_{44}^{\beta}$ has also increased (compared to the spherical case, refer Table 5) as a result of freeing the aspect ratio.

Fig. 13 presents the posterior distribution of the anisotropy ratio, shear modulus $\left(\mu^{\prime}\right)$, and bulk modulus (K). No well-defined peak is seen for the anisotropy ratio, multiple values between (3 - 9) show significant probabilities. These values are however much higher than the values for spherical inclusion (Fig. 10). The theoretical bulk modulus $\mathrm{K}$ can be calculated from experimental macroscopic Young's modulus assuming a Poisson's ratio of 0.36 , $K=\frac{E}{3(1-2 v)}$, equals to $K=83 \mathrm{GPa}$, which is in line with the predicted value: $\mathrm{K}=88 \mathrm{GPa}$. The bulk modulus is also closer to the value ( $100 \mathrm{GPa})$ suggested by the first-principle analysis of Ti-V binary alloys. The shear modulus $\left(\mu^{\prime}\right)$ peaks at $\sim 5.6 \mathrm{GPa}$, which is also closer to Raghunathan et al. [14] observation for Ti-1023 alloy with similar microstructure as the current study. The ensemble of the $C_{i j}^{\beta}$ constants identified by Raghunathan et al. [14] lies within the Bayesian identified distribution.

The correlation coefficients of the multivariate distribution of anisotropy ratio, bulk modulus, and shear modulus for the ellipsoid case are presented in Table 8. A moderate positive correlation is seen between the bulk modulus and the shear modulus.

Fig. 14 shows the experimental and simulated data for the identified $C_{i j}^{\beta}$ and $\beta_{A R}$. By varying the aspect ratio of the $\beta$ phase, a better agreement is achieved with the transverse lattice strain of the $\beta$ phase, which was not seen with the previous case. Overall, allowing the aspect ratio of the $\beta$ phase as a free variable in the optimization process improves the fitting and thereby minimizing the uncertainty on the identification of the SEC of the $\beta$ phase.

It should also be noted that simulations with a higher number of iterations ( $\sim 1$ million) were also carried out, but the results were similar. Bayesian inference of the $C_{i j}^{\beta}$ with sphere and prolate morphology presents two different trends.

For most $\beta$-type titanium alloys, the $\mu^{\prime}$ ranges from 10 to $30 \mathrm{GPa}$ and the $\mu^{\prime \prime}$ from 35 to $45 \mathrm{GPa}$ (refer to Table 1 ). In the case of the sphere morphology, the shear modulus $\left(\mu^{\prime}=\sim 17 \mathrm{GPa}\right)$ was within the literature range while the $\mu^{\prime \prime}=\sim 29 \mathrm{GPa}$ was lower 

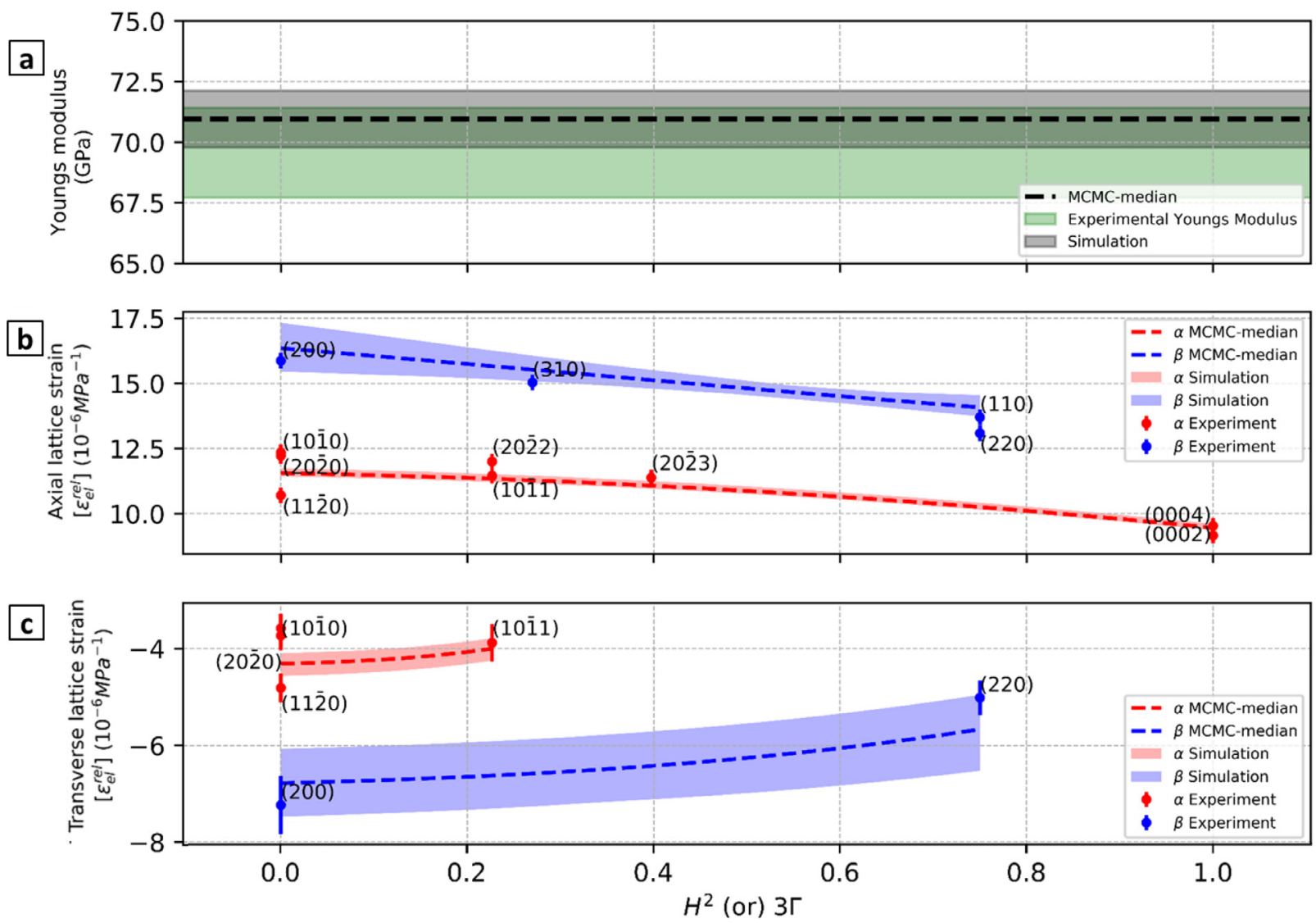

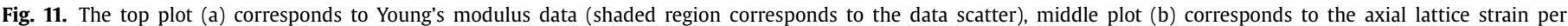

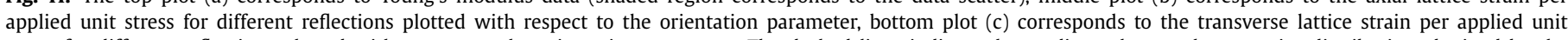

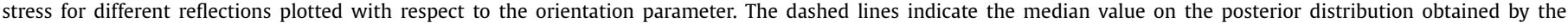
MCMC simulation, and the shaded region indicates the three standard deviations.

Table 8

Correlation coefficient of the multivariate distribution presented in Fig. 13.

\begin{tabular}{llll}
\hline & Anisotropy ratio $(\mathrm{A})$ & Bulk modulus $(\mathrm{K})$ & Shear modulus $\left(\mu^{\prime}\right)$ \\
\hline Anisotropy ratio $(\mathrm{A})$ & 1 & -0.001 & -0.001 \\
Bulk modulus $(\mathrm{K})$ & -0.001 & 1 & 0.42 \\
Shear modulus $\left(\mu^{\prime}\right)$ & -0.027 & 0.42 & 1.00 \\
\hline
\end{tabular}

than most of the $\beta$ Ti-alloys. While for the prolate morphology, an inverse trend was seen with $\mu^{\prime \prime}=\sim 43 \mathrm{GPa}$ value being well within the literature range and the $\mu^{\prime}=\sim 5.6 \mathrm{GPa}$ being lower than most of the $\beta$ Ti-alloys.

\section{Discussion}

\subsection{In-situ XRD data bias}

The lattice strains of different (hkl) reflections strongly affect the choice of the identified SEC, especially, when the analysis itself is concerned with the uncertainty measurement. For instance, obtaining precise lattice strains values require careful processing of the 2D XRD data. Conventional data analysis is a multi-step process from detector calibration to peak fitting, which is prone to errors. Here, we have tried to minimize this by including only the non-overlapping (hkl) reflections with good intensities. Due to the large material parameter space probed, to be efficient only the axial and transverse reflections were considered for the fitting. But in theory, we should be able to use the complete in-plane strains and even a complete strain pole-figure to reduce the uncertainty on the identified elastic constants. In the case of well-defined and separate peaks, one could even employ the 2D XRD-DIC technique [83] to analyze the complete in-plane strains with quite a good accuracy.

In the present study, the grain shape is found to be an important factor during the identification process. Especially it improved the fitting on the transverse lattice strains. The transverse reflections show a higher degree of peak asymmetry and lower intensity and hence the standard error for some of the transverse reflections is higher than their axial counterparts. For example, the (200) axial peak comes only from all the (100) aligned axial grains whose normal is aligned with the tensile axis, while the (200) transverse peak can have a contribution from (100) and (110) axially aligned grains, and more generally all (hk0) grains. The axial behavior of (100) is representative of one-grain family (100), while the transverse behavior is an average of all (hk0) grain families. This leads to dispersion in the transverse lattice strains which in turn leads to higher standard deviation in the identified elastic constants. However, such effects should be minimized in the current study due to the diverse experimental dataset used to calculate the average lattice strains. The effect of texture on the identified SEC is further detailed in section Vc. The higher standard deviations in the identified SEC can be further addressed by defining weights of individual 


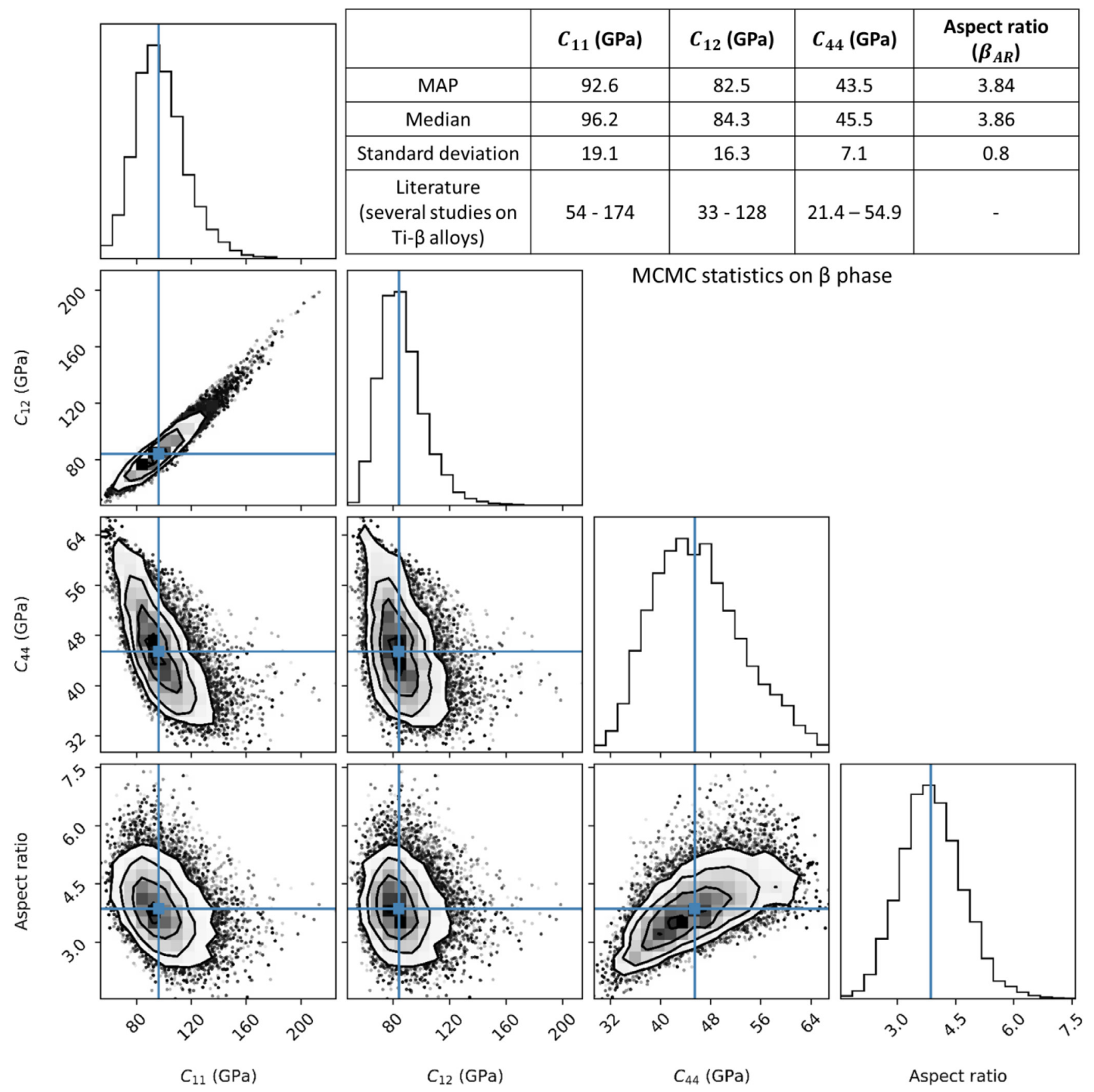

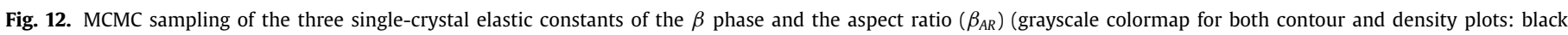
representing high probability regions and gray representing lower probability region).

(hkl) reflection proportional to the integral area of the (hkl) peak in XRD spectra. Alongside, other aspects of the XRD data such as the FWHM could also be used to gauge the stress/strain asymmetry, if required.

The discrepancy between the literature value of $C_{44}^{\beta}$ and the one identified from MCMC distribution could possibly stem from the experimental measurement errors (in the XRD or stiffness analysis, and as well as the experimental techniques employed in the literature). Similar disagreement in the $C_{44}^{\beta}$ was also seen in the case of SEC identified from indentation modulus of BCC Fe-3\%Si alloy [45]. It was suggested that this could be improved by identifying specific reflections in the experimental dataset that could refine the $C_{44}^{\beta}$ estimate. It should also be noted that the litera- ture identified SEC for $\beta$ Ti-alloy vary both in chemistry as well as in microstructure. Hence, trying to find any correlation among the current identified values and the literature ones may not be fruitful.

\section{2. $\beta$ phase stability of Ti-1023}

In literature, it is reported that for the $\beta$-type titanium alloys, the shear modulus $\left(\mu^{\prime}\right)$ dictates the stability of the $\beta$ phase and can be adjusted by the $\beta$-stabilizing elements. It was also reported that a lower value of $\mu^{\prime}$ in BCC indicates increased instability with respect to shear on $\{110\}$ plane along $\langle 1 \overline{1} 0\rangle$, which results in a martensitic transformation [18]. This is the case especially here 

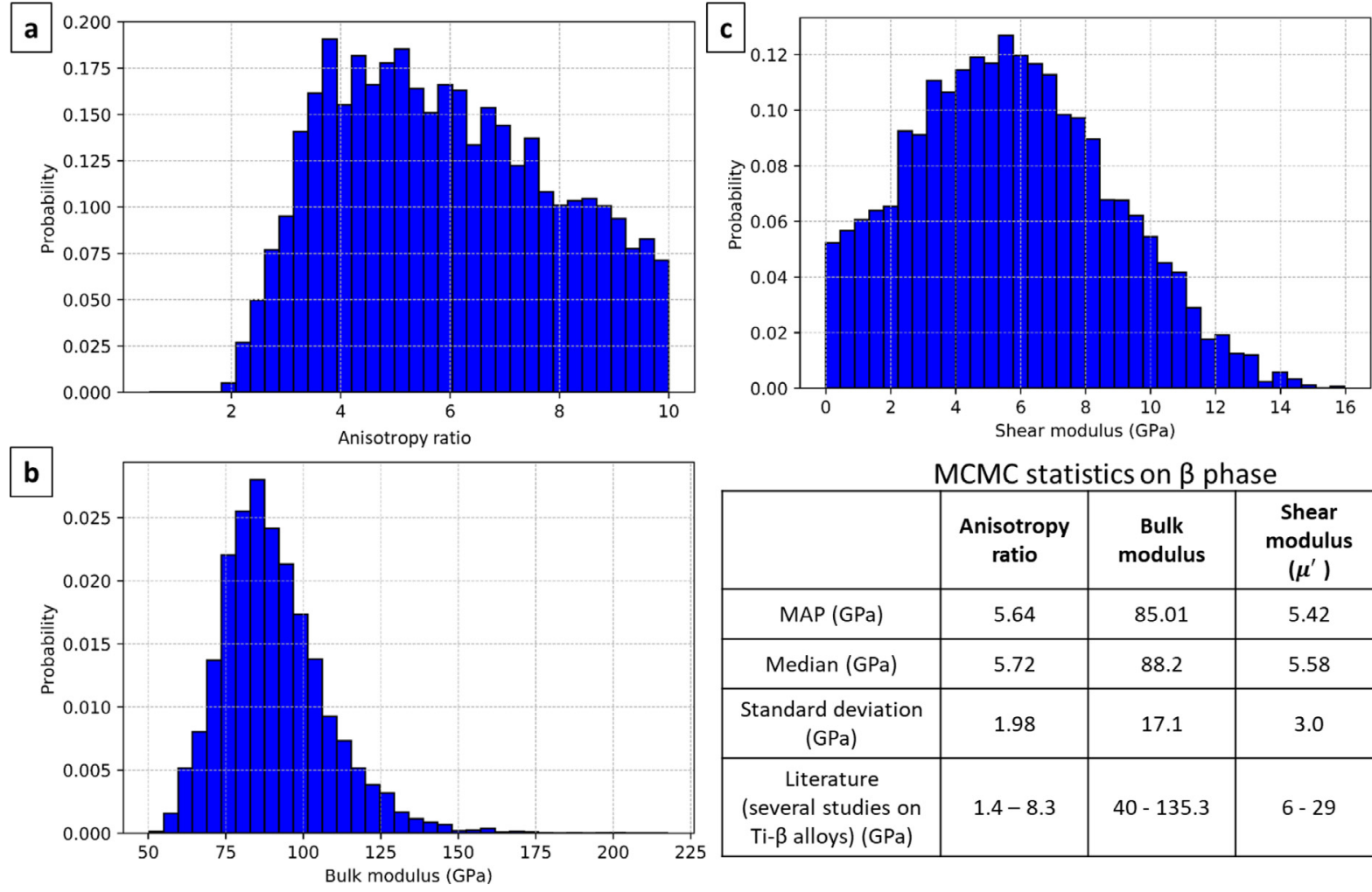

\begin{tabular}{|c|c|c|c|}
\hline & $\begin{array}{c}\text { Anisotropy } \\
\text { ratio }\end{array}$ & $\begin{array}{c}\text { Bulk } \\
\text { modulus }\end{array}$ & $\begin{array}{c}\text { Shear } \\
\text { modulus } \\
\left(\boldsymbol{\mu}^{\prime}\right)\end{array}$ \\
\hline MAP (GPa) & 5.64 & 85.01 & 5.42 \\
\hline Median (GPa) & 5.72 & 88.2 & 5.58 \\
\hline $\begin{array}{c}\text { Standard deviation } \\
\text { (GPa) }\end{array}$ & 1.98 & 17.1 & 3.0 \\
\hline $\begin{array}{c}\text { Literature } \\
\text { (several studies on } \\
\text { Ti- } \beta \text { alloys) (GPa) }\end{array}$ & $1.4-8.3$ & $40-135.3$ & $6-29$ \\
\hline
\end{tabular}

Fig. 13. MCMC sampling of the anisotropy ratio $\mathrm{A}$, bulk (K) and shear $\left(\mu^{\prime}\right)$ moduli of the $\beta$ phase.
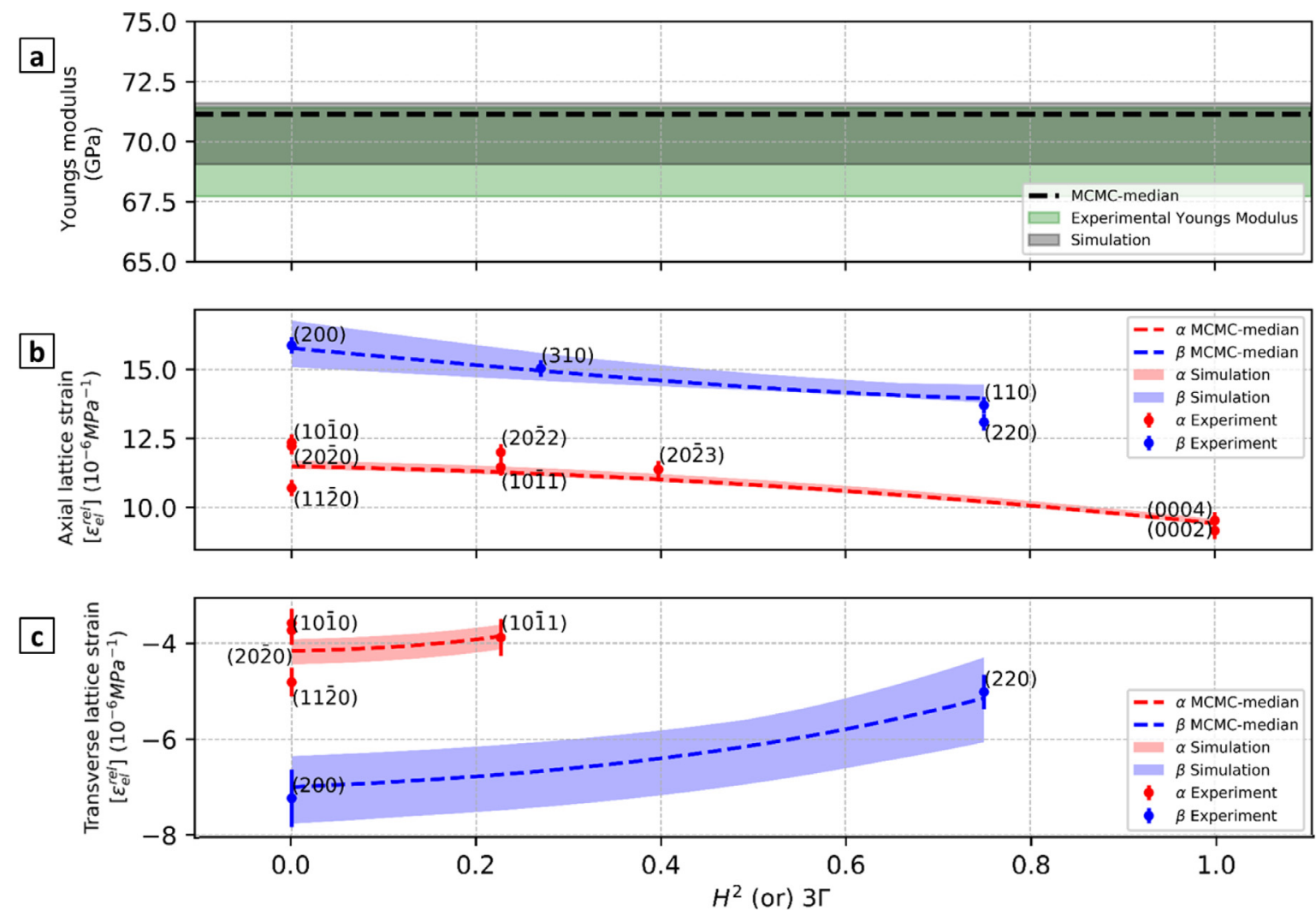

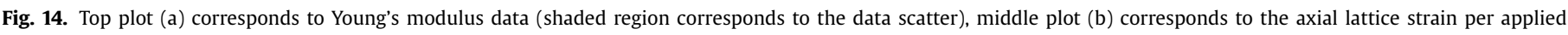

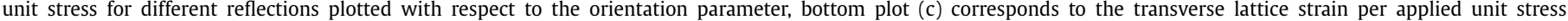

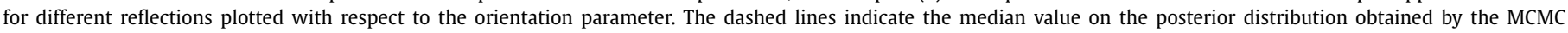
simulation, and the shaded region indicates the three standard deviations. 

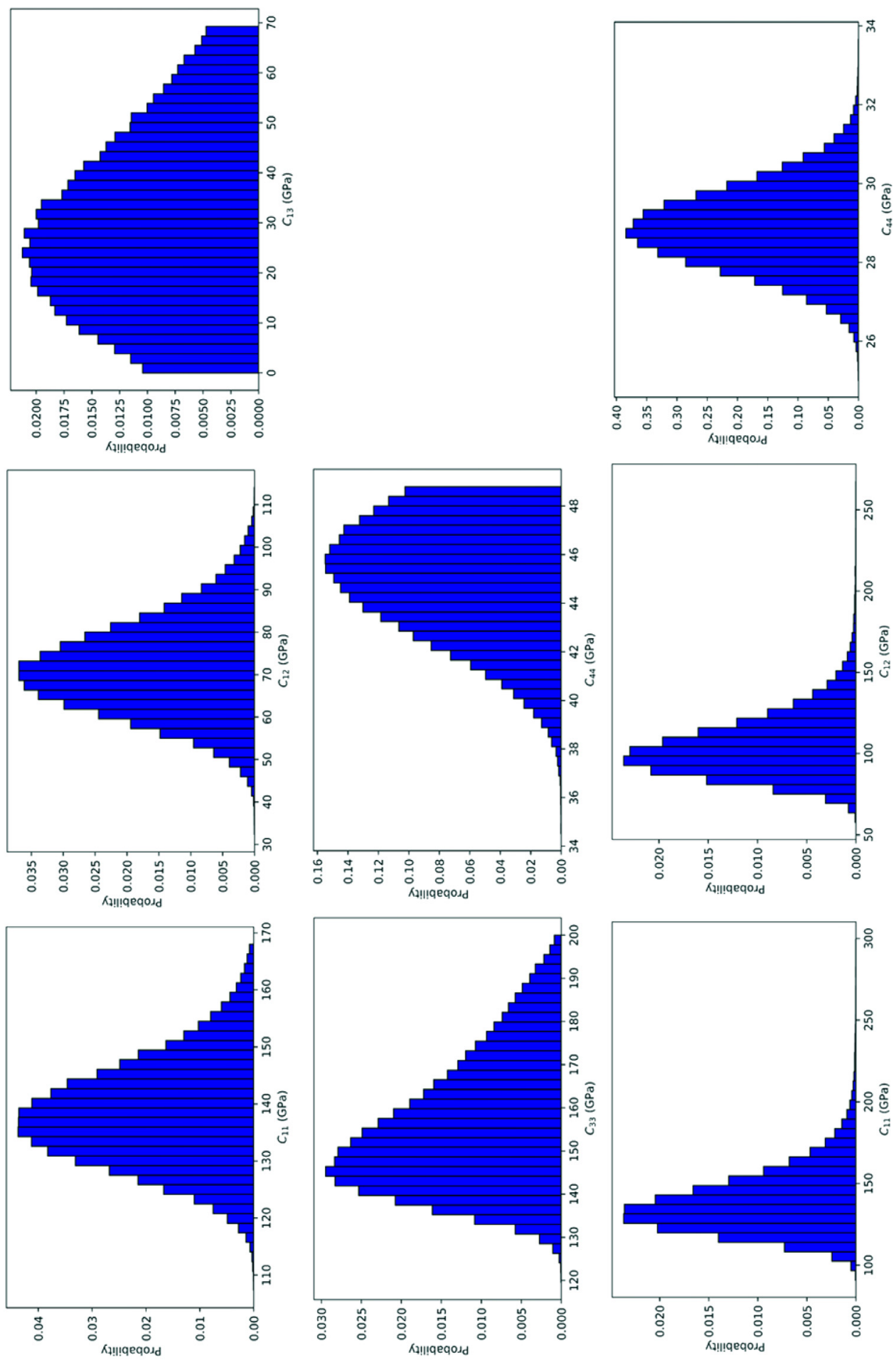

$\pi$

Fig. 15. MCMC sampling of the (a) five SECs of the $\alpha$ phase and (b) three SECs of the $\beta$ phase.

where a martensitic transformation is seen at stresses above 400 MPa. This was also verified with the Bayesian inference (for prolate spheroid) suggesting a low shear modulus ( $\left.\mu^{\prime} \sim 5.6 \mathrm{GPa}\right)$ for the available experimental data. Here, the softening of the shear modulus $\mu^{\prime}$ or $\mu^{\prime \prime}$ still needs to be explained. The difference in one of the shear modulus $\left(\mu^{\prime \prime}\right)$ can stem from the chemical composition of the alloy as suggested by the first-principles studies on $\operatorname{Ti}_{\mathrm{X}} \mathrm{V}_{1-\mathrm{x}} \mathrm{C}$ [84], wherein the shear modulus, Young's modulus, and hardness seem to be proportionally increasing with the $\mathrm{V}$ concentration. A study on low Young's modulus Ti-39Nb [15] alloy suggested the polycrystalline Young's modulus is proportional to the shear moduli $\left(\mu^{\prime \prime}\right.$ and $\left.\mu^{\prime}\right)$. Here, for the as-forged Ti-1023 the Young's modulus ( $\sim 70 \mathrm{GPa}$ ) is lower than most of the $\beta$ Ti-alloys in the literature. Consequently, one can argue that the lower value of $\mu^{\prime}$ or $\mu^{\prime \prime}$ modulus could be a direct consequence of the macroscopic Young's modulus. 
Table 9

MCMC statistics on the posterior distribution of $C_{\mathrm{ij}}^{\beta}$ with spherical grains having experimental and random texture.

\begin{tabular}{|c|c|c|c|c|c|c|}
\hline & \multicolumn{2}{|l|}{$C_{11}$} & \multicolumn{2}{|l|}{$C_{12}$} & \multicolumn{2}{|l|}{$C_{44}$} \\
\hline & Experimental. texture & Random texture & Experimental texture & Random texture & Experimental texture & Random texture \\
\hline MAP (GPa) & 134.3 & 141.08 & 101.5 & 106.13 & 28.8 & 27.85 \\
\hline Median (GPa) & 142.6 & 147.93 & 109 & 114.09 & 28.9 & 27.86 \\
\hline Standard deviation (GPa) & 24.3 & 27.12 & 24.5 & 27.37 & 1.13 & 0.98 \\
\hline
\end{tabular}

Table 10

Available range of values supplied for the prior variable in MCMC sampling.

\begin{tabular}{lllllllll}
\hline & $C_{11}^{\beta}$ & $C_{12}^{\beta}$ & $C_{44}^{\beta}$ & $C_{11}^{\alpha}$ & $C_{12}^{\alpha}$ & $C_{13}^{\alpha}$ & $C_{33}^{\alpha}$ & $C_{44}^{\alpha}$ \\
\hline Elastic moduli range (GPa) & $70-500$ & $60-500$ & $25-60$ & $100-168$ & $20-115$ & $1-70$ & $100-200$ & $30-50$ \\
\hline
\end{tabular}

Table 11

Correlation coefficients of the multivariate distribution presented in Fig. 15.

\begin{tabular}{lllllllll}
\hline & $C_{11}^{\beta}$ & $C_{12}^{\beta}$ & $C_{44}^{\beta}$ & $C_{11}^{\alpha}$ & $C_{12}^{\alpha}$ & $C_{13}^{\alpha}$ & $C_{33}^{\alpha}$ & $C_{44}^{\alpha}$ \\
\hline$C_{11}^{\beta}$ & 1.0 & 0.997 & -0.506 & -0.303 & -0.359 & -0.077 & -0.018 & 0.044 \\
$C_{12}^{\beta}$ & 0.997 & 1 & -0.444 & -0.304 & -0.36 & -0.078 & -0.019 & 0.047 \\
$C_{44}^{\beta}$ & -0.506 & -0.444 & 1 & 0.125 & 0.174 & 0.034 & -0.004 & -0.076 \\
$C_{11}^{\alpha}$ & -0.303 & -0.304 & 0.125 & 1 & 0.937 & 0.236 & 0.163 & -0.134 \\
$C_{12}^{\alpha}$ & -0.359 & -0.36 & 0.174 & 0.937 & 1 & -0.022 & -0.08 & 0.079 \\
$C_{13}^{\alpha}$ & -0.077 & -0.078 & 0.034 & 0.236 & -0.022 & 1 & 0.93 & -0.554 \\
$C_{33}^{\alpha}$ & -0.018 & -0.019 & -0.004 & 0.163 & -0.08 & 0.93 & 1 & -0.528 \\
$C_{44}^{\alpha}$ & 0.044 & 0.047 & -0.076 & -0.134 & 0.079 & -0.554 & -0.528 & 1 \\
\hline
\end{tabular}

Table 12

MCMC statistics on the posterior distribution of $C_{\mathrm{ij}}^{\beta}$ and $\mathrm{C}_{\mathrm{ij}}^{\alpha}$.

\begin{tabular}{lllllllll}
\hline & $C_{11}^{\beta}$ & $C_{12}^{\beta}$ & $C_{44}^{\beta}$ & $C_{11}^{\alpha}$ & $C_{12}^{\alpha}$ & $C_{13}^{\alpha}$ & $C_{33}^{\alpha}$ & \multicolumn{1}{c}{$C_{44}^{\alpha}$} \\
\hline MAP (GPa) & 131.4 & 98.2 & 28.8 & 135.6 & 71.1 & 24.1 & 145.7 & 45.7 \\
Median (GPa) & 135.8 & 101.89 & 28.8 & 137.75 & 70.98 & 28.5 & 153.17 & 45.12 \\
Standard deviation (GPa) & 19.4 & 19.5 & 1.04 & 9.05 & 10.87 & 17.01 & 14.87 & 2.36 \\
Literature range (GPa) & $97.7-175$ & $70-128$ & $36-55$ & $160-168$ & $90-114.5$ & $66-69.3$ & $181-191$ & $38-48.8$ \\
\hline
\end{tabular}

\subsection{Effect of $\alpha$ and $\beta$ crystallographic textures on the MCMC sampling}

It is recalled that in the current study, the tested samples did not show a very sharp texture for both the $\alpha$ and $\beta$ phase. To analyze the impact of texture on the SEC identification, a random texture was generated for both the $\alpha$ and $\beta$ phases (as in [23]), and was employed during the MCMC sampling. A total of 250,000 iterations (with 150,000 iterations to tune the variance) were carried out with spherical morphologies for the grains. The results of the random texture analysis are presented in Table 9 along with the case of experimental texture. It is observed that texture does not influence the identified SEC significantly. They are practically the same within their uncertainties. The results are in line with the fact that the (hkl) lattice strains in textured material do not vary by more than few percent of the (hkl) lattice strains in a texture free aggregate [85].

\subsection{Influence of the $\alpha$ phase on the MCMC sampling}

In all previous cases, the SEC of the $\alpha$ phase has been fixed because there is agreement on the SEC in literature. The effect of SEC of the $\alpha$ phase on the MCMC sampling is considered while keeping spherical grain shape for both $\alpha$ and $\beta$ phases, thereby equation (8) is now a function of 8 free variables.

$\frac{\varepsilon_{\text {hkl }}^{\text {simulation }}}{\Sigma_{33}^{\max }}=f\left(C_{11}^{\beta}, C_{12}^{\beta}, C_{44}^{\beta}, C_{11}^{\alpha}, C_{12}^{\alpha}, C_{13}^{\alpha}, C_{33}^{\alpha}, C_{44}^{\alpha}\right)$

Since the number of free variables is higher ( 5 for the $\alpha$ phase and 3 for the $\beta$ phase), subsequently the number of MCMC iterations were also increased to 2 million (with 1 million iterations to tune the variance of the proposal distribution) to explore a large space range of $C_{i j}^{\beta \alpha}$. The range of prior probed during MCMC sampling is presented in Table 10.

Fig. 15 shows the multivariate posterior distribution of the $C_{i j}^{\beta}$ and $C_{i j}^{\alpha}$. The correlation coefficients of the multivariate distribution $\left(\mathrm{C}_{\mathrm{ij}}^{\beta}\right.$ and $\left.\mathrm{C}_{i j}^{\alpha}\right)$ are presented in Table 11 . The strong correlation between $C_{11}^{\beta}$ and $C_{12}^{\beta}$ is still maintained, additionally positive correlation can also be seen between $C_{11}^{\alpha}$ and $C_{12}^{\alpha}$ and between $C_{33}^{\alpha}$ and $\mathrm{C}_{13}^{\alpha}$.

The MCMC statistics of the posterior distribution is provided in Table 12. Interestingly, allowing the $C_{i j}^{\alpha}$ as a free variable has no significant effect on the $C_{i j}^{\beta}$ posterior distribution. Small changes in the median and standard deviation of $C_{11}^{\beta}$ and $C_{12}^{\beta}$ are seen, while the $\left(C_{11}^{\beta}-C_{12}^{\beta}\right)$ remains rather constant. There is no agreement on the MCMC identified $C_{i j}^{\alpha}$ values with the literature (except for the $C_{44}^{\alpha}$ constant). The wider distribution of some of the $C_{i j}^{\alpha}$ constants $\left(C_{13}^{\alpha}, C_{33}^{\alpha}\right)$ suggest low sensitivity of the changes to the experimental data. This could also be due to the low volume fraction of the $\alpha$ phase (15\%). No strong difference is observed in the posterior distribution of the $C_{i j}^{\beta}$ when the $C_{i j}^{\alpha}$ is considered as a free or fixed variable. This leads to a possible conclusion that the $C_{i j}^{\alpha}$ have a negligible effect on $\mathrm{C}_{\mathrm{ij}}^{\beta}$ uncertainty quantification at the volume fraction of $15 \%$.

Subsequently, the volume fraction of the $\alpha$ phase is also checked for its influence on the MCMC sampling. The volume fraction of the $\alpha$ phase ranges between $12 \%$ to $16 \%$ with an average of $15 \%$ (SEM image analysis and Rietveld refinement of the HEXRD 
Table 13

The available range of values supplied for the prior variable in MCMC sampling.

\begin{tabular}{llllll}
\hline & $C_{11}^{\beta}$ & $C_{12}^{\beta}$ & $C_{44}^{\beta}$ & $\mathrm{f}_{\alpha}$ & $\mathrm{f}_{\beta}$ \\
\hline Range & $70-500 \mathrm{GPa}$ & $60-500 \mathrm{GPa}$ & $25-60 \mathrm{GPa}$ & $0.12 \%-0.16 \%$ & $\left(1-\mathrm{f}_{\alpha}\right)$ \\
\hline
\end{tabular}

Table 14

MCMC statistics on the posterior distribution of $C_{\mathrm{ij}}^{\beta}$ with $\mathrm{f}_{\alpha}$ as a fixed and free variable.

\begin{tabular}{|c|c|c|c|c|c|c|}
\hline & \multicolumn{2}{|l|}{$C_{11}$} & \multicolumn{2}{|l|}{$C_{12}$} & \multicolumn{2}{|l|}{$C_{44}$} \\
\hline & $\begin{array}{l}\text { Fixed volume } \\
\text { fraction }\left(\mathrm{f}_{\alpha}=15 \%\right)\end{array}$ & $\begin{array}{l}\text { Volume fraction } \\
\left(\mathrm{f}_{\alpha}=12-16 \%(\right.\end{array}$ & $\begin{array}{l}\text { Fixed volume } \\
\text { fraction }\left(f_{\alpha}=15 \%\right)\end{array}$ & $\begin{array}{l}\text { Volume fraction } \\
\left(\mathrm{f}_{\alpha}=12-16 \%\right)\end{array}$ & $\begin{array}{l}\text { Fixed volume } \\
\text { fraction }\left(\mathrm{f}_{\alpha}=15 \%\right)\end{array}$ & $\begin{array}{l}\text { Volume fraction } \\
\left(\mathrm{f}_{\alpha}=12-16 \%\right)\end{array}$ \\
\hline MAP (GPa) & 134.3 & 134.3 & 101.5 & 100.4 & 28.8 & 29.2 \\
\hline Median (GPa) & 142.6 & 144.3 & 109 & 110.3 & 28.9 & 29.3 \\
\hline Standard deviation (GPa) & 24.3 & 24.5 & 24.5 & 24.7 & 1.13 & 1.15 \\
\hline
\end{tabular}

pattern). A total of 500,000 iterations (with 350,000 iterations to tune the variance) were carried out with priors having uniform distribution, see Table 13.

No significant changes are observed in the distribution of $C_{i j}^{\beta}$ when the volume fraction is considered as a free variable. This suggests that the range $(12-16 \%)$ in the measurement of the volume fraction of the $\alpha$ phase has no significant effect on the posterior distribution of the $C_{i j}^{\beta}$ constants, refer to Table 14 .

In all the above discussion cases, when the morphology of the grains is changed from sphere to prolate, the resulting distribution changes. This suggests that in any combination of free variables in equation (8), the morphology (aspect ratio) of the $\beta$-phase has a significant effect on the resulting posterior distribution.

\section{Conclusions}

An elastic self-consistent scheme (ELSC) for anisotropic polycrystals combined with high energy X-ray diffraction was employed in a Bayesian framework for in-situ mechanical tests to extract the single-crystal elastic constants (SEC) of the $\beta$ phase of a near- $\beta$ titanium alloy, Ti-1023. The as-forged state of the Ti1023 alloy is characterized by Young's modulus of $70 \mathrm{GPa}$ and a strength of around $800 \mathrm{MPa}$. Moreover, at stress higher than $400 \mathrm{MPa}$, the formation of stress-induced martensite was clearly revealed by XDR spectra, which demonstrates the low $\beta$-phase stability. The in-situ XRD, due to its phase selectivity, allows evaluating the SEC in a multiphase material. The ELSC model was used to predict the (hkl) specific elastic strains and macroscopic stiffness and was then compared with the XRD (hkl) specific lattice strains and experimental Young's modulus. The versatility of the Bayesian framework was evaluated on the Ti-1023 with an as-forged microstructure. We have systematically investigated the effect of the different intrinsic parameters of the multi-phase material to identify and study their effect on the MCMC sampling of the $\beta$ phase SEC. The results of this investigation are as follows:

- the SEC for the cubic $\beta$ phase was extracted first in a framework with spherical grains $\left(C_{11}^{\beta}=134.3 \pm 24.3 \mathrm{GPa}, \quad C_{12}^{\beta}=\right.$ $\left.101.5 \pm 24.5 \mathrm{GPa}, \quad C_{44}^{\beta}=28.8 \pm 1.13 \mathrm{GPa}\right)$. The constant $C_{11}^{\beta}$ and $C_{12}^{\beta}$ were found to be in good agreement with the constants of the pure $\beta$ phase alloy in the literature, but presented highest relative uncertainty, while the $C_{44}^{\beta}$ constant has the lowest uncertainty (magnitude is within $10 \%$ of the literature). The shear modulus $\mu^{\prime}=\frac{C_{11}-C_{12}}{2}$ was within the literature range, while the other shear modulus $\mu^{\prime \prime}=C_{44}^{\beta}$ modulus had a comparatively lower value.

- The grain morphology parameter of the $\beta$ phase $\left(\beta_{A R}\right)$ resulted in a significant effect on the MCMC sampling of the $\beta$ phase SEC. The identified SEC $\left(C_{11}^{\beta}=92.6 \pm 19.1 \mathrm{GPa}, \quad C_{12}^{\beta}=\right.$ $\left.82.5 \pm 16.3 \mathrm{GPa}, \quad C_{44}^{\beta}=43.5 \pm 7.1 \mathrm{GPa}\right)$ and the aspect ratio $\beta_{A R}=3.8 \pm 0.8$ results in a distinct bulk modulus, shear modulus coherent with the experimental observations and literature range. The shear modulus $\mu^{\prime}$ was much lower than the literature range, while the $\mu^{\prime \prime}$ modulus agreed well with the literature. Also, a wide range of anisotropy ratio (3-10), in agreement with the literature scatter, was found to satisfy the experimental data set.

- Texture, volume fraction of the $\alpha$ phase, and even the SEC of the $\alpha\left(\mathrm{C}_{\mathrm{ij}}^{\alpha}\right)$ phase have been found to have an insignificant effect on the identification of the SEC of the $\beta$ phase for the current experimental dataset, within the texture and volume fraction variations investigated here. It is however worth noting that, more generally, the interplay between crystallographic texture, grain morphology, grain arrangement, and elastic response is a complex and non-intuitive topic that demands testing a large number of different combinations (see for example [86]) and it has therefore been left for future studies.

However, certain local phenomena such as clustering of orientations, morphological orientations, or a phase embedded in a specific environment cannot be analyzed with the standard selfconsistent scheme. A future approach would be to couple the Bayesian inference with full-field micromechanical modeling, e.g. based on the Fast Fourier transform (FFT) method [87] to probe different architectures, local environments and spatial arrangements of grains.

\section{Data availability}

The datasets generated and analyzed during the current study are available from the corresponding author on reasonable request.

\section{Declaration of Competing Interest}

The authors declare that they have no known competing financial interests or personal relationships that could have appeared to influence the work reported in this paper.

\section{Acknowledgments}

This research is supported by the "Région Grand Est" and by the French State through two programs operated by the National Research Agency (ANR), (1) "Investment in the future" referenced by ANR-11-LABX-0008-01 (Laboratory of Excellence "DAMAS": Design of Alloy Metals for low-mAss Structures) and (2) "Plan d'Investissement d'Avenir" (PIA) in the frame of a research program managed by "Institut de Recherche Technologique Matériaux, Métallurgie, Procédés" (IRT M2P). We acknowledge DIAMOND for provision of synchrotron radiation beamtime at beamline I12-JEEP. 
The authors also thank the Laboratoire Léon Brillouin (France) for beamtime allocation and Sebastien GAUTROT (LLB, France) for his help during the experiments. Vincent Jacquemain, Dr. Jean-Baptiste Marijon, and Dr. Stefan Michalik are acknowledged for their help during the synchrotron campaign at Diamond.

\section{References}

[1] R.R. Boyer, R.D. Briggs, The use of $\beta$ titanium alloys in the aerospace industry, J. Mater. Eng. Perform. 14 (2005) 681-685

[2] M.R. Chini, L. Germain, N. Gey, S. Andrieu, T. Duval, Advanced Microtexture Analysis of A Ti 10-2-3 Product for Better Understanding of Local Variations in Mechanical Behavior, in: Proc. 13th World Conf. Titan, Wiley Online Library, 2016, pp. 1943-1948.

[3] C. Zener, Contributions to the theory of beta-phase alloys, Phys. Rev. 71 (1947) 846.

[4] A. Heldmann, M. Hoelzel, M. Hofmann, W. Gan, W.W. Schmahl, E. Griesshaber, T. Hansen, N. Schell, W. Petry, Diffraction-based determination of single-crystal elastic constants of polycrystalline titanium alloys, J. Appl. Crystallogr. (2019) 52.

[5] S. Fréour, D. Gloaguen, M. François, A. Perronnet, R. Guillén, Determination of single-crystal elasticity constants in a cubic phase within a multiphase alloy: X-ray diffraction measurements and inverse-scale transition modelling, J. Appl. Crystallogr. 38 (2005) 30-37.

[6] S. Fréour, E. Lacoste, M. François, R. Guillén, Determining Ti-17 $\beta$-phase single-crystal elasticity constants through X-ray diffraction and inverse scale transition model, Mater. Sci. Forum, Trans Tech Publ (2011) 97-102.

[7] J. Nejezchlebová, M. Janovská, H. Seiner, P. Sedlák, M. Landa, J. Šmilauerová, J. Stráský, P. Harcuba, M. Janeček, The effect of athermal and isothermal $\omega$ phase particles on elasticity of $\beta$-Ti single crystals, Acta Mater 110 (2016) 185-191.

[8] G. Martin, Simulation numérique multi-échelles du comportement mécanique des alliages de titane bêta-métastable Ti5553 et Ti17, (2012).

[9] W. Petry, A. Heiming, J. Trampenau, M. Alba, C. Herzig, H.R. Schober, G. Vogl, Phonon dispersion of the bcc phase of group-IV metals. I. bcc titanium, Phys. Rev. B. 43 (1991) 10933.

[10] E.A. Brandes, G.B. Brook, Smithells Metal Ref. Book, Butterwarth, 1992.

[11] E.S. Fisher, D. Dever, Relation of the $c^{\prime}$ elastic modulus to stability of bcc transition metals, Acta Metall 18 (1970) 265-269.

[12] J.-Y. Kim, S.I. Rokhlin, Determination of elastic constants of generally anisotropic inclined lamellar structure using line-focus acoustic microscopy, J. Acoust. Soc. Am. 126 (2009) 2998-3007.

[13] H. Ledbetter, H. Ogi, S. Kai, S. Kim, M. Hirao, Elastic constants of body-centered-cubic titanium monocrystals, J. Appl. Phys. 95 (2004) 4642-4644.

[14] S.L. Raghunathan, A.M. Stapleton, R.J. Dashwood, M. Jackson, D. Dye, Micromechanics of Ti-10V-2Fe-3Al: In situ synchrotron characterisation and modelling, Acta Mater 55 (2007) 6861-6872.

[15] Q. Meng, J. Zhang, Y. Huo, Y. Sui, J. Zhang, S. Guo, X. Zhao, Design of low modulus $\beta$-type titanium alloys by tuning shear modulus C44, J. Alloys Compd. 745 (2018) 579-585

[16] R.J. Talling, R.J. Dashwood, M. Jackson, S. Kuramoto, D. Dye, Determination of (C11-C12) in Ti-36Nb-2Ta-3Zr-0.3 O (wt.\%)(Gum metal), Scr. Mater. 59 (2008) 669-672.

[17] Q. Meng, Q. Liu, S. Guo, Y. Zhu, X. Zhao, Effect of thermo-mechanical treatment on mechanical and elastic properties of Ti-36Nb-5Zr alloy, Prog. Nat. Sci. Mater. Int. 25 (2015) 229-235.

[18] E.G. Obbard, Y.L. Hao, R.J. Talling, S.J. Li, Y.W. Zhang, D. Dye, R. Yang, The effect of oxygen on $\alpha$ "martensite and superelasticity in Ti-24Nb-4Zr-8Sn, Acta Mater 59 (2011) 112-125.

[19] J.J. Bhattacharyya, S. Nair, D.C. Pagan, V. Tari, A.D. Rollett, S.R. Agnew, In-situ high energy X-ray diffraction study of the elastic response of a metastable $\beta$-titanium alloy, Acta Mater (2020)

[20] E.G. Obbard, Y.L. Hao, T. Akahori, R.J. Talling, M. Niinomi, D. Dye, R. Yang, Mechanics of superelasticity in Ti-30Nb-(8-10) Ta-5Zr alloy, Acta Mater. 58 (2010) 3557-3567.

[21] M. Tane, T. Nakano, S. Kuramoto, M. Hara, M. Niinomi, N. Takesue, T. Yano, H. Nakajima, Low Young's modulus in Ti-Nb-Ta-Zr-O alloys: Cold working and oxygen effects, Acta Mater 59 (2011) 6975-6988.

[22] H.W. Jeong, Y.S. Yoo, Y.T. Lee, J.K. Park, Elastic softening behavior of Ti-Nb single crystal near martensitic transformation temperature, J. Appl. Phys. 108 (2010) 63515

[23] S. Lhadi, R.R.P. Purushottam Raj Purohit, T. Richeton, N. Gey, S. Berbenni, O. Perroud, L. Germain, Elasto-viscoplastic tensile behavior of as-forged Ti-1023 alloy: Experiments and micromechanical modeling, Mater. Sci. Eng. A. (2020) 139491.

[24] S. Lhadi, M.-R. Chini, T. Richeton, N. Gey, L. Germain, S. Berbenni, Micromechanical Modeling of the Elasto-Viscoplastic Behavior and Incompatibility Stresses of $\beta$-Ti Alloys, Materials (Basel) 11 (2018) 1227.

[25] M. Abdel-Hady, K. Hinoshita, M. Morinaga, General approach to phase stability and elastic properties of $\beta$-type Ti-alloys using electronic parameters, Scr. Mater. 55 (2006) 477-480.

[26] B.E. Tegner, L. Zhu, G.J. Ackland, Relative strength of phase stabilizers in titanium alloys, Phys. Rev. B. 85 (2012) 214106.
[27] Q.-M. Hu, S.-J. Li, Y.-L. Hao, R. Yang, B. Johansson, L. Vitos, Phase stability and elastic modulus of Ti alloys containing $\mathrm{Nb}, \mathrm{Zr}$, and/or $\mathrm{Sn}$ from first-principles calculations, Appl. Phys. Lett. 93 (2008) 121902.

[28] S. Lhadi, S. Berbenni, N. Gey, T. Richeton, L. Germain, Micromechanical modeling of the effect of elastic and plastic anisotropies on the mechanical behavior of $\beta$-Ti alloys, Int. J. Plast. 109 (2018) 88-107.

[29] J.F. Nye, Physical properties of crystals: their representation by tensors and matrices, Oxford university press, 1985.

[30] D.H. Chung, W.R. Buessem, The elastic anisotropy of crystals, J. Appl. Phys. 38 (1967) 2010-2012.

[31] K. Tanaka, M. Koiwa, Single-crystal elastic constants of intermetallic compounds, Intermetallics 4 (1996) S29-S39.

[32] S. Matthies, H.G. Priesmeyer, M.R. Daymond, On the diffractive determination of single-crystal elastic constants using polycrystalline samples, J. Appl. Crystallogr. 34 (2001) 585-601.

[33] J.R. Cho, D. Dye, K.T. Conlon, M.R. Daymond, R.C. Reed, Intergranular strain accumulation in a near-alpha titanium alloy during plastic deformation, Acta Mater 50 (2002) 4847-4864

[34] W. Voigt, Lehrbuch der kristallphysik, Teubner Leipzig, 1928.

[35] A. Reuss, Calculation of the flow limits of mixed crystals on the basis of the plasticity of monocrystals, Z. Angew. Math. Mech. 9 (1929) 49-58.

[36] J.D. Eshelby, The determination of the elastic field of an ellipsoidal inclusion, and related problems, Proc. R. Soc. London. Ser. A. Math. Phys. Sci. 241 (1957) 376-396.

[37] E. Kröner, Berechnung der elastischen Konstanten des Vielkristalls aus den Konstanten des Einkristalls, Zeitschrift Für Phys 151 (1958) 504-518.

[38] A. Hershey, The elasticity of an isotropic aggregate of anisotropic cubic crystals, J. Appl. Mech. ASME. 21 (1954) 236-240.

[39] R. Hill, A self-consistent mechanics of composite materials, J. Mech. Phys. Solids. 13 (1965) 213-222.

[40] J.W. Hutchinson, Elastic-plastic behaviour of polycrystalline metals and composites, Proc. R. Soc. London. A. Math. Phys. Sci. 319 (1970) 247-272.

[41] D. Gloaguen, G. Oum, V. Legrand, J. Fajoui, M.-J. Moya, T. Pirling, W. Kockelmann, Intergranular strain evolution in titanium during tensile loading: neutron diffraction and polycrystalline model, Metall. Mater. Trans. A. 46 (2015) 5038-5046.

[42] R.R.P. Purushottam Raj Purohit, S. Lhadi, N. Gey, O. Castelnau, T. Richeton, L. Germain, S. Berbenni, Stress partitioning in a near- $\beta$ Titanium alloy induced by elastic and plastic phase anisotropies: experimental and modeling, 14th World Conf. Titan, 2019.

[43] A.M. Stapleton, S.L. Raghunathan, I. Bantounas, H.J. Stone, T.C. Lindley, D. Dye, Evolution of lattice strain in Ti-6Al-4V during tensile loading at room temperature, Acta Mater 56 (2008) 6186-6196.

[44] D. Dye, H.J. Stone, R.C. Reed, Intergranular and interphase microstresses, Curr. Opin. Solid State Mater. Sci. 5 (2001) 31-37.

[45] A.R. Castillo, S.R. Kalidindi, A Bayesian Framework for the Estimation of the Single Crystal Elastic Parameters from Spherical Indentation Stress-Strain Measurements, Front. Mater. 6 (2019) 136.

[46] M.W. Priddy, Exploration of forward and inverse protocols for property optimization of Ti-6Al-4V, (2016)

[47] D.K. Patel, H.F. Al-Harbi, S.R. Kalidindi, Extracting single-crystal elastic constants from polycrystalline samples using spherical nanoindentation and orientation measurements, Acta Mater 79 (2014) 108-116.

[48] A.R. Castillo, V.R. Joseph, S.R. Kalidindi, Bayesian sequential design of experiments for extraction of single-crystal material properties from spherical indentation measurements on polycrystalline samples, JOM 71 (2019) 2671-2679.

[49] C. Andrieu, N. De Freitas, A. Doucet, M.I. Jordan, An introduction to MCMC for machine learning, Mach. Learn. 50 (2003) 5-43.

[50] M.D. Abràmoff, P.J. Magalhães, S.J. Ram, Image processing with ImageJ, Biophotonics Int 11 (2004) 36-42.

[51] B. Beausir, J.J. Fundenberger, Analysis tools for electron and X-ray diffraction, ATEX-Software, Univ. Lorraine-Metz, 2017.

[52] M. Drakopoulos, T. Connolley, C. Reinhard, R. Atwood, O. Magdysyuk, N. Vo M. Hart, L. Connor, B. Humphreys, G. Howell, I12: the joint engineering, environment and processing (JEEP) beamline at diamond light source, J. Synchrotron Radiat. 22 (2015) 828-838.

[53] I. Astm, ASTM E8/E8M-16a: Standard Test Methods for Tension Testing of Metallic Materials, ASTM Int., West Conshohocken, PA, USA, 2016.

[54] T.W. Duerig, G.T. Terlinde, J.C. Williams, Phase transformations and tensile properties of Ti-10V-2Fe-3AI, Metall. Trans. A. 11 (1980) 1987-1998.

[55] T.W. Duerig, J. Albrecht, D. Richter, P. Fischer, Formation and reversion of stress induced martensite in Ti-10V-2Fe-3Al, Acta Met 30 (1982) 2161-2172.

[56] T.W. Duerig, J.C. Williams, Overview: microstructure and properties of beta titanium alloys, Beta Titan. Alloy. 1980's (1983) 19-67.

[57] C. Li, X. Wu, J.H. Chen, S. van der Zwaag, Influence of $\alpha$ morphology and volume fraction on the stress-induced martensitic transformation in Ti-10V-2Fe-3Al, Mater. Sci. Eng. A. 528 (2011) 5854-5860.

[58] A. Borbely, L. Renversade, P. Kenesei, J. Wright, On the calibration of high-energy X-ray diffraction setups. I. Assessing tilt and spatial distortion of the area detector, J. Appl. Crystallogr. 47 (2014) 1042-1053.

[59] A. Borbély, L. Renversade, P. Kenesei, On the calibration of high-energy X-ray diffraction setups. II. Assessing the rotation axis and residual strains, J. Appl. Crystallogr. 47 (2014) 1585-1595

[60] B.B. He, Two-dimensional X-ray diffraction, Wiley Online Library, 2009. 
[61] F.G. Zhang, M. Bornert, J. Petit, O. Castelnau, Accuracy of stress measurement by Laue microdiffraction (Laue-DIC method): the influence of image noise, calibration errors and spot number, J. Synchrotron Radiat. 24 (2017) 802-817.

[62] J. Kieffer, D. Karkoulis, PyFAI, a versatile library for azimuthal regrouping, J. Phys. Conf. Ser (2013) 36.

[63] M. Wojdyr, Fityk: a general-purpose peak fitting program, J. Appl. Crystallogr. 43 (2010) 1126-1128.

[64] R.A. Lebensohn, C.N. Tomé, P.P. Castaneda, Self-consistent modelling of the mechanical behaviour of viscoplastic polycrystals incorporating intragranular field fluctuations, Philos. Mag. 87 (2007) 4287-4322.

[65] L.J. Walpole, On the overall elastic moduli of composite materials, J. Mech. Phys. Solids. 17 (1969) 235-251.

[66] M. Berveiller, O. Fassi-Fehri, A. Hihi, The problem of two plastic and heterogeneous inclusions in an anisotropic medium, Int. J. Eng. Sci. 25 (1987) 691-709.

[67] C. Mareau, S. Berbenni, An affine formulation for the self-consistent modeling of elasto-viscoplastic heterogeneous materials based on the translated field method, Int. J. Plast. 64 (2015) 134-150.

[68] R.A. Lebensohn, P.P. Castañeda, R. Brenner, O. Castelnau, Full-field vs. homogenization methods to predict microstructure-property relations for polycrystalline materials, in: Comput. Methods Microstruct. Relationships, Springer, 2011, pp. 393-441.

[69] R. Brenner, R.A. Lebensohn, O. Castelnau, Elastic anisotropy and yield surface estimates of polycrystals, Int. J. Solids Struct. 46 (2009) 3018-3026.

[70] R.A. Lebensohn, Y. Liu, P.P. Castaneda, On the accuracy of the self-consistent approximation for polycrystals: comparison with full-field numerical simulations, Acta Mater 52 (2004) 5347-5361.

[71] H. Rappel, L.A.A. Beex, S.P.A. Bordas, Bayesian inference to identify parameters in viscoelasticity, Mech. Time-Dependent Mater. 22 (2018) 221-258.

[72] H. Rappel, L.A.A. Beex, J.S. Hale, L. Noels, S.P.A. Bordas, A tutorial on bayesian inference to identify material parameters in solid mechanics, Arch. Comput. Methods Eng. 27 (2020) 361-385.

[73] H. Rappel, L.A.A. Beex, L. Noels, S.P.A. Bordas, Identifying elastoplastic parameters with Bayes' theorem considering output error, input error and model uncertainty, Probabilistic Eng. Mech. 55 (2019) 28-41.

[74] H. Rappel, L.A.A. Beex, Estimating fibres' material parameter distributions from limited data with the help of Bayesian inference, Eur. J. Mech. 75 (2019) 169-196.
[75] T. Bayes LII, An essay towards solving a problem in the doctrine of chances. By the late Rev. Mr. Bayes, FRS communicated by Mr. Price, in a letter to John Canton, AMFR S, Philos. Trans. R. Soc. London. (1763) 370-418.

[76] J.L. Beck, S.-K. Au, Bayesian updating of structural models and reliability using Markov chain Monte Carlo simulation, J. Eng. Mech. 128 (2002) 380-391.

[77] R.F.S. Hearmon, The elastic constants of crystals and other anisotropic materials, Landolt-Bornstein Tables, III/18 (1984) 1154.

[78] F. Mouhat, F.-X. Coudert, Necessary and sufficient elastic stability conditions in various crystal systems, Phys. Rev. B. 90 (2014) 224104.

[79] C. Fonnesbeck, A. Patil, D. Huard, J. Salvatier, PyMC: Bayesian stochastic modelling in Python, Ascl (2015) ascl-1506.

[80] P. Virtanen, R. Gommers, T.E. Oliphant, M. Haberland, T. Reddy, D. Cournapeau, E. Burovski, P. Peterson, W. Weckesser, J. Bright, SciPy 1.0: fundamental algorithms for scientific computing in Python, Nat. Methods. 17 (2020) 261-272.

[81] M.E. Fitzpatrick, A. Lodini, Analysis of residual stress by diffraction using neutron and synchrotron radiation, CRC Press, 2003.

[82] L. Germain, M.R. Bache, Crystallographic texture and the definition of effective structural unit size in titanium products, Ti-2007, in: Sci. Technol., 2007, pp. 953-956. Niinomi, M., Akiyama, S., Hagiwara, M., Ikeda, M., Maruyama, K., Eds..

[83] H. Zhang, T. Sui, E. Salvati, D. Daisenberger, A.J.G. Lunt, K.S. Fong, X. Song, A.M. Korsunsky, Digital image correlation of 2D X-ray powder diffraction data for lattice strain evaluation, Materials (Basel) 11 (2018) 427.

[84] B. Wang, Y. Liang, Y. Liu, J. Ye, Electronic and Elastic Properties of TIXV1XC: First-principles Calculations, in: Adv. Mater. Eng. Proc. 2015 Int. Conf. Adv. Mater. Eng., World Scientific, 2016, pp. 131-140.

[85] H.F. Poulsen, Introduction to the characterization of residual stress by neutron diffraction, MT Hutchings, PJ Withers, TM Holden, T. Lorentzen, Taylor\&Francis, London (2005), 401 pp., Price£ 49.99 (Hardback), ISBN: 0-415-31000-8, (2007).

[86] T. Gu, O. Castelnau, S. Forest, E. Hervé-Luanco, F. Lecouturier, H. Proudhon, L. Thilly, Multiscale modeling of the elastic behavior of architectured and nanostructured $\mathrm{Cu}-\mathrm{Nb}$ composite wires, Int. J. Solids Struct. 121 (2017) $148-162$.

[87] H. Moulinec, P. Suquet, A FFT-based numerical method for computing the mechanical properties of composites from images of their microstructures, in: IUTAM Symp. Microstruct. Interact. Compos. Mater., Springer, 1995, pp. 235-246. 